quatrième série-tome 41 fascicule 6 novembre-décembre 2008

$$
\begin{aligned}
& \text { ANNALES } \\
& \text { SCIENTIFIQUES } \\
& \text { de } \\
& \text { L'ECOLE } \\
& \text { NORMALE } \\
& \text { SUPÉRIEURE }
\end{aligned}
$$

Viktor PETROV \& Nikita SEMENOV \& Kirill ZAINOULLINE J-imvariant of linear algebraic groups 


\title{
$J$-INVARIANT OF LINEAR ALGEBRAIC GROUPS
}

\author{
BY VikTor PETROV, Nikita SEMENOV \\ AND Kirill ZAINOULLINE
}

\begin{abstract}
Let $G$ be a semisimple linear algebraic group of inner type over a field $F$, and let $X$ be a projective homogeneous $G$-variety such that $G$ splits over the function field of $X$. We introduce the $J$-invariant of $G$ which characterizes the motivic behavior of $X$, and generalizes the $J$-invariant defined by A. Vishik in the context of quadratic forms.

We use this $J$-invariant to provide motivic decompositions of all generically split projective homogeneous $G$-varieties, e.g. Severi-Brauer varieties, Pfister quadrics, maximal orthogonal Grassmannians, varieties of Borel subgroups of $G$. We also discuss relations with torsion indices, canonical dimensions and cohomological invariants of the group $G$.
\end{abstract}

RÉSUMÉ. - Soit $G$ un groupe algébrique linéaire semi-simple de type intérieur sur un corps $F$ et soit $X$ un $G$-espace homogène projectif tel que le groupe $G$ soit déployé sur le point générique de $X$. Nous introduisons le $J$-invariant de $G$ qui caractérise le comportement motivique de $X$ et généralise le $J$-invariant défini par A. Vishik dans le cadre des formes quadratiques.

Nous utilisons cet invariant pour obtenir les décompositions motiviques de tous les $G$-espaces homogènes projectifs qui sont génériquement déployés, par exemple les variétés de Severi-Brauer, les quadriques de Pfister, la grassmannienne des sous-espaces totalement isotropes maximaux d'une forme quadratique, la variété des sous-groupes de Borel de $G$. Nous discutons également les relations avec les indices de torsion, la dimension canonique et les invariants cohomologiques du groupe $G$.

\section{Introduction}

Let $G$ be a semisimple linear algebraic group over a field $F$, and let $X$ be a projective homogeneous $G$-variety. We are interested in direct sum decomposition of the GrothendieckChow motive $\mathcal{M}(X)$ of $X$.

Motivic decompositions are fundamental mathematical tools which in recent years have led to the resolution of several classical problems. For instance, the motivic decomposition of a Pfister quadric plays a major role in Voevodsky's proof of the Milnor conjecture. A proof 
of the generalization of this conjecture known as the Bloch-Kato conjecture has been announced by Rost and Voevodsky, and uses in an essential way the motivic decompositions of norm varieties which are closely related to projective homogeneous varieties.

As another application of motivic decompositions we mention Vishik's [36] recent breakthrough concerning the Kaplansky problem, where Vishik uses the notion of a $J$-invariant of an orthogonal group. In fact, our paper has been motivated by Vishik's work, and the $J$-invariant we introduce is a generalization to an arbitrary semisimple algebraic group.

Concerning decompositions of motives of homogenous $G$-varieties $X$ it was first observed by Köck [25] that if the group $G$ is split (i.e. contains a split maximal torus), the motive of $X$ is isomorphic to a direct sum of twisted Tate motives, thus has the simplest possible decomposition. Chernousov-Gille-Merkurjev [7] and Brosnan [4] proved that if $G$ is isotropic (i.e. contains a split 1-dimensional torus), the motive of $X$ decomposes as a direct sum of motives of projective homogeneous varieties of smaller dimensions corresponding to anisotropic groups, which reduces the problem to the anisotropic case. For anisotropic groups only a few partial results are known. In this case the components of a motivic decomposition of $X$ are generally expected to be of a non-geometric nature, i.e. not the (twisted) motives of some other varieties. The first examples of such decompositions were provided by Rost [32] who proved that the motive of a Pfister quadric decomposes as a direct sum of twisted copies of a so-called Rost motive $\mathcal{R}$, which is a priori non-geometric. The motives of Severi-Brauer varieties have been computed by Karpenko [21]. For examples of motivic decompositions of exceptional varieties, see Bonnet [2] (varieties of type $\mathrm{G}_{2}$ ), and Nikolenko-Semenov-Zainoulline [30] (varieties of type $\mathrm{F}_{4}$ ). Note that in all these examples the group $G$ splits over the generic point of $X$; we will call such varieties generically split.

Our main result is a uniform proof of the above results. We show (see Theorem 5.17):

MAIN THeOREM. - Let $G$ be a semisimple linear algebraic group of inner type over a field $F$ and let $p$ be a prime integer. Let $X$ be a generically split projective homogeneous $G$-variety. Then the Chow motive of $X$ with $\mathbb{Z} / p$-coefficients is isomorphic to a direct sum

$$
\mathcal{M}(X ; \mathbb{Z} / p) \simeq \bigoplus_{i \in \mathcal{I}} \mathcal{R}_{p}(G)(i)
$$

of twisted copies of an indecomposable motive $\mathcal{R}_{p}(G)$ for some finite multiset $\mathcal{I}$ of non-negative integers.

Observe that the motive $\mathcal{R}_{p}(G)$ depends on $G$ and $p$ but not on the type of a parabolic subgroup defining $X$. Moreover, considered with $\mathbb{Q}$-coefficients $\mathcal{R}_{p}(G)$ always splits as a direct sum of twisted Tate motives.

Our proof has two key ingredients. The first is the Rost Nilpotence Theorem proved for projective quadrics by Rost, and generalized to arbitrary projective homogeneous varieties by Brosnan [4] and Chernousov-Gille-Merkurjev [7]. Roughly speaking, this result plays the role of the Galois descent for motivic decompositions over a separable closure $\bar{F}$ of $F$, and reduces the problem to the description of idempotent cycles in the endomorphism group $\operatorname{End}\left(\mathcal{M}\left(X_{\bar{F}} ; \mathbb{Z} / p\right)\right)$ which are defined over $F$. The second key point in our proof comes from the topology of compact Lie groups. In [20] Kac invented the notion of $p$-exceptional degrees, which are numbers that relate the degrees of mod $p$ basic polynomial invariants 
with the $p$-torsion part of the Chow ring of a compact Lie group. Zainoulline [38] has shown that these numbers describe the generic part of the subgroup of cycles in $\operatorname{End}\left(\mathcal{M}\left(X_{\bar{F}} ; \mathbb{Z} / p\right)\right)$ defined over $F$.

To describe the whole subgroup we introduce the $J$-invariant of a group $G \bmod p$, which is a list of non-negative integers denoted by $J_{p}(G)$ (see Definition 4.6). In most cases the values of $J_{p}(G)$ are implicitly computed in [20] and can easily be extracted from Table 4.13. The $J$-invariant measures the 'size' of the motive $\mathcal{R}_{p}(G)$ and in this way characterizes the motivic decomposition of $X$. For instance, if the $J$-invariant takes its minimal possible non-trivial value $J_{p}(G)=(1)$, then the motive $\mathcal{R}_{p}(G) \otimes \mathbb{Q}$ has the following recognizable decomposition (cf. $[37, \S 5]$ and $[33, \S 5])$

$$
\mathcal{R}_{p}(G) \otimes \mathbb{Q} \simeq \bigoplus_{i=0}^{p-1} \mathbb{Q}\left(i \cdot \frac{p^{n-1}-1}{p-1}\right), \text { where } n=2 \text { or } 3 .
$$

The assignment $G \mapsto \mathcal{R}_{p}(G)$ can be viewed as a motivic analogue of the cohomological invariant of $G$ given by the Tits class of $G$ if $n=2$, and by the Rost invariant of $G$ if $n=3$. In these cases the motive $\mathcal{R}_{p}(G)$ coincides with a generalized Rost motive.

We also generalize some of the results in [5]. Using the motivic version of the main result of Edidin-Graham [12] on cellular fibrations we provide a general formula which expresses the motive of the total space of a cellular fibration in terms of the motives of its base (see Theorem 3.7). We also provide several criteria for the existence of liftings of motivic decompositions via the reduction map $\mathbb{Z} \rightarrow \mathbb{Z} / m$, and prove such liftings always exist (see Theorem 2.16).

The paper is organized as follows. In the first section we provide several auxiliary facts concerning motives and rational cycles. The rather technical Section 2 is devoted to lifting of idempotents. In Section 3 we discuss the motives of cellular fibrations. The next section is devoted to the notion of a $J$-invariant. The proof of the main result is given in Section 5. The last two sections are devoted to various applications of the $J$-invariant and examples of motivic decompositions. In particular, we discuss the relations with canonical $p$-dimensions, degrees of zero-cycles, and the Rost invariant.

\section{Chow motives and rational cycles}

In the present section we follow the notation and definitions from [13, Ch. XII] (see also [26]).

Let $X$ be a smooth projective irreducible variety over a field $F$. Let $\mathrm{CH}_{i}(X ; \Lambda)$ be the Chow group of cycles of dimension $i$ on $X$ with coefficients in a commutative ring $\Lambda$. For simplicity we denote $\mathrm{CH}(X ; \mathbb{Z})$ by $\mathrm{CH}(X)$.

1.1. Definition. - Following [13, §63] an element $\phi \in \mathrm{CH}_{\operatorname{dim} X+d}(X \times Y ; \Lambda)$ is called a correspondence between $X$ and $Y$ of degree $d$ with coefficients in $\Lambda$. Let $\phi \in \mathrm{CH}(X \times$ $Y ; \Lambda)$ and $\psi \in \mathrm{CH}(Y \times Z ; \Lambda)$ be correspondences of degrees $d$ and $e$ respectively. Then their product $\psi \circ \phi$ is defined by the formula $\left(\operatorname{pr}_{X Z}\right)_{*}\left(\operatorname{pr}_{X Y}^{*}(\phi) \cdot \operatorname{pr}_{Y Z}^{*}(\psi)\right)$ and has degree $d+e$. The correspondence product endows the group $\mathrm{CH}(X \times X ; \Lambda)$ with a ring structure. The identity element of this ring is the class of the diagonal $\Delta_{X}$. Given $\phi \in \mathrm{CH}(X \times X ; \Lambda)$ of degree $d$ we define a $\Lambda$-linear map $\mathrm{CH}_{i}(X ; \Lambda) \rightarrow \mathrm{CH}_{i+d}(Y ; \Lambda)$ by $\alpha \mapsto\left(\operatorname{pr}_{Y}\right)_{*}\left(\operatorname{pr}_{X}^{*}(\alpha) \cdot \phi\right)$. This map 
is called realization of $\phi$ and is denoted by $\phi_{\star}$. By definition $(\psi \circ \phi)_{\star}=\psi_{\star} \circ \phi_{\star}$. Given a correspondence $\phi$ we denote its transpose by $\phi^{t}$.

1.2. Definition. - Following $[13, \S 64]$ let $\mathcal{M}(X ; \Lambda)$ denote the Chow motive of $X$ with $\Lambda$-coefficients and let $\mathcal{M}(X ; \Lambda)(n)=\mathcal{M}(X ; \Lambda) \otimes \Lambda(n)$ be the respective $t w i s t$ by the Tate motive. For simplicity we will denote $\mathcal{M}(X ; \mathbb{Z})$ by $\mathcal{M}(X)$. Recall that morphisms between $\mathcal{M}(X ; \Lambda)(n)$ and $\mathcal{M}(Y ; \Lambda)(m)$ are given by correspondences of degree $n-m$. The group of endomorphisms $\operatorname{End}(\mathcal{M}(X ; \Lambda))$ coincides with the Chow group $\mathrm{CH}_{\operatorname{dim} X}(X \times X ; \Lambda)$. Observe that to provide a direct sum decomposition of $\mathcal{M}(X ; \Lambda)$ is the same as to provide a family of pair-wise orthogonal idempotents $\phi_{i} \in \operatorname{End}(\mathcal{M}(X ; \Lambda))$ such that $\sum_{i} \phi_{i}=\Delta_{X}$.

1.3. Definition. - Assume that a motive $M$ is a direct sum of twisted Tate motives. In this case its Chow group $\mathrm{CH}(M)$ is a free abelian group. We define its Poincaré polynomial as

$$
P(M, t)=\sum_{i \geq 0} a_{i} t^{i}
$$

where $a_{i}$ is the rank of $\mathrm{CH}_{i}(M)$.

1.4. Definition. - Let $L / F$ be a field extension. We say $L$ is a splitting field of a smooth projective variety $X$ or, equivalently, a variety $X$ splits over $L$ if the motive $\mathcal{M}(X ; \mathbb{Z})$ is isomorphic over $L$ to a finite direct sum of twisted Tate motives.

1.5. ExAmple. - A variety $X$ over a field $F$ is called cellular if $X$ has a proper descending filtration by closed subvarieties $X_{i}$ such that each complement $X_{i} \backslash X_{i+1}$ is a disjoint union of affine spaces defined over $F$. According to [13, Corollary 66.4] if $X$ is cellular, then $X$ splits over $F$.

In particular, let $G$ be a semisimple linear algebraic group over a field $F$ and let $X$ be a projective homogeneous $G$-variety. Assume that the group $G$ splits over the generic point of $X$, i.e. $G_{F(X)}=G \times{ }_{F} F(X)$ contains a split maximal torus defined over $F(X)$. Then $X_{F(X)}$ is a cellular variety and, therefore, $F(X)$ is a splitting field of $X$. Some concrete examples of such varieties are provided in 3.6.

1.6. Definition. - Assume $X$ has a splitting field $L$. We will write $\mathrm{CH}(\bar{X} ; \Lambda)$ for $\mathrm{CH}\left(X_{L} ; \Lambda\right)$ and $\overline{\mathrm{CH}}(X ; \Lambda)$ for the image of the restriction map $\mathrm{CH}(X ; \Lambda) \rightarrow \mathrm{CH}(\bar{X} ; \Lambda)$ (cf. $[22,1.2])$. Similarly, we denote by $\mathcal{M}(\bar{X} ; \Lambda)$ the motive of $X$ considered over $L$. If $M$ is a direct summand of $\mathcal{M}(X ; \Lambda)$, we denote by $\bar{M}$ the motive $M_{L}$. The elements of $\overline{\mathrm{CH}}(X ; \Lambda)$ will be called rational cycles on $X_{L}$ with respect to the field extension $L / F$ and the coefficient ring $\Lambda$. If $L^{\prime}$ is another splitting field of $X$, then there is a chain of canonical isomorphisms $\mathrm{CH}\left(X_{L}\right) \simeq \mathrm{CH}\left(X_{L L^{\prime}}\right) \simeq \mathrm{CH}\left(X_{L^{\prime}}\right)$, where $L L^{\prime}$ is the composite of $L$ and $L^{\prime}$. Hence, the groups $\mathrm{CH}(\bar{X})$ and $\overline{\mathrm{CH}}(X)$ do not depend on the choice of $L$.

1.7. - According to [22, Remark 5.6] there is the Künneth decomposition $\mathrm{CH}(\bar{X} \times$ $\bar{X})=\mathrm{CH}(\bar{X}) \otimes \mathrm{CH}(\bar{X})$ and Poincaré duality holds for $\mathrm{CH}(\bar{X})$. The latter means that given a basis of $\mathrm{CH}(\bar{X})$ there is a dual one with respect to the non-degenerate pairing $(\alpha, \beta) \mapsto \operatorname{deg}(\alpha \cdot \beta)$, where deg is the degree map. In view of the Künneth decomposition the correspondence product of cycles in $\mathrm{CH}(\bar{X} \times \bar{X})$ is given by the formula 
$\left(\alpha_{1} \times \beta_{1}\right) \circ\left(\alpha_{2} \times \beta_{2}\right)=\operatorname{deg}\left(\alpha_{1} \beta_{2}\right)\left(\alpha_{2} \times \beta_{1}\right)$, the realization by $(\alpha \times \beta)_{\star}(\gamma)=\operatorname{deg}(\alpha \gamma) \beta$ and the transpose by $(\alpha \times \beta)^{t}=\beta \times \alpha$.

Sometimes we will use the contravariant notation $\mathrm{CH}^{*}$ for Chow groups meaning $\mathrm{CH}^{i}(X)=\mathrm{CH}_{\operatorname{dim} X-i}(X)$ for irreducible $X$. The following important fact will be used in the proof of the main theorem (see Lemma 5.7).

1.8. Lemma. - Let $X$ and $Y$ be two smooth projective varieties such that $Y$ is irreducible, $F(Y)$ is a splitting field of $X$ and $Y$ has a splitting field. For any $r$ consider the projection in the Künneth decomposition

$$
\operatorname{pr}_{0}: \mathrm{CH}^{r}(\bar{X} \times \bar{Y})=\bigoplus_{i=0}^{r} \mathrm{CH}^{r-i}(\bar{X}) \otimes \mathrm{CH}^{i}(\bar{Y}) \rightarrow \mathrm{CH}^{r}(\bar{X}) .
$$

Then for any $\rho \in \mathrm{CH}^{r}(\bar{X})$ we have $\operatorname{pr}_{0}^{-1}(\rho) \cap \overline{\mathrm{CH}^{r}}(X \times Y) \neq \varnothing$.

Proof. - Let $L$ be a common splitting field of $X$ and $Y$. The lemma follows from the commutative diagram

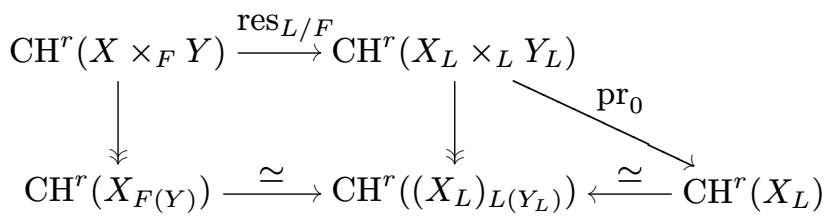

where the left square is obtained by taking the generic fiber of the base change morphism $X_{L} \rightarrow X$; the vertical arrows are taken from the localization sequence for Chow groups and, hence, are surjective; and the bottom horizontal maps are isomorphisms since $L$ is a splitting field.

1.9. Definition. - We say that a field extension $E / F$ is rank preserving with respect to $X$ if the restriction map $\operatorname{res}_{E / F}: \mathrm{CH}(X) \rightarrow \mathrm{CH}\left(X_{E}\right)$ becomes an isomorphism after tensoring with $\mathbb{Q}$.

1.10. Lemma. - Assume $X$ has a splitting field. Then for any rank preserving finite field extension $E / F$ we have $[E: F] \cdot \overline{\mathrm{CH}}\left(X_{E}\right) \subset \overline{\mathrm{CH}}(X)$.

Proof. - Let $L$ be a splitting field containing $E$. Let $\gamma$ be any element in $\overline{\mathrm{CH}}\left(X_{E}\right)$. By definition there exists $\alpha \in \mathrm{CH}\left(X_{E}\right)$ such that $\gamma=\operatorname{res}_{L / E}(\alpha)$. Since $\operatorname{res}_{E / F} \otimes \mathbb{Q}$ is an isomorphism, there exists an element $\beta \in \mathrm{CH}(X)$ and a non-zero integer $n$ such that $\operatorname{res}_{E / F}(\beta)=n \alpha$. By the projection formula

$$
n \cdot \operatorname{cores}_{E / F}(\alpha)=\operatorname{cores}_{E / F}\left(\operatorname{res}_{E / F}(\beta)\right)=[E: F] \cdot \beta .
$$

Applying $\operatorname{res}_{L / E}$ to the both sides of the identity we obtain

$$
n\left(\operatorname{res}_{L / E}\left(\operatorname{cores}_{E / F}(\alpha)\right)\right)=n[E: F] \cdot \gamma .
$$

Therefore, $\operatorname{res}_{L / E}\left(\operatorname{cores}_{E / F}(\alpha)\right)=[E: F] \cdot \gamma$.

We provide now examples of varieties for which any field extension is rank preserving and, hence, Lemma 1.10 holds. 
1.11. Definition. - Let $G$ be a semisimple linear algebraic group over a field $F$ and let $X$ be a projective homogeneous $G$-variety. Let $\mathcal{D}$ denote the Dynkin diagram of $G$. According to [34] one can always choose a quasi-split group $G_{0}$ over $F$ with the same Dynkin diagram, a parabolic subgroup $P$ of $G_{0}$ and a cocycle $\xi \in Z^{1}\left(F, G_{0}\right)$ such that $G$ is isogenous to the twisted form ${ }_{\xi} G_{0}$ and $X$ is isomorphic to ${ }_{\xi}\left(G_{0} / P\right)$. If $G_{0}$ is split (see 1.5), then $G$ is called a group of inner type over $F$.

1.12. Lemma. - Let $G$ be a semisimple linear algebraic group of inner type over a field $F$ and let $X$ be a projective homogeneous $G$-variety. Then any field extension $E / F$ is rank preserving with respect to $X$ and $X \times X$.

Proof. - By [31, Theorem 2.2 and 4.2] the restriction map $K_{0}(X) \rightarrow K_{0}\left(X_{E}\right)$ becomes an isomorphism after tensoring with $\mathbb{Q}$. Since the Chern character

$$
\text { ch: } K_{0}(X) \otimes \mathbb{Q} \rightarrow \mathrm{CH}^{*}(X) \otimes \mathbb{Q}
$$

is an isomorphism and respects pull-backs, $E$ is rank preserving with respect to $X$. It remains to note that $X \times X$ is a homogeneous $G \times G$-variety.

1.13. Remark. - For even dimensional quadrics with non-trivial discriminant the restriction map $K_{0}(X) \rightarrow K_{0}(\bar{X})$ is not surjective and Lemma 1.12 does not hold.

\section{Lifting of idempotents}

This section is devoted to lifting of idempotents and isomorphisms. First, we treat the case of general graded algebras. The main results here are Lemma 2.5 and Proposition 2.6. Then, assuming Rost Nilpotence 2.8 we provide conditions to lift motivic decompositions and isomorphisms (Theorem 2.16).

2.1. Definition. - Let $A^{*}$ be a $\mathbb{Z}$-graded ring. Assume we are given two orthogonal idempotents $\phi_{i}$ and $\phi_{j}$ in $A^{0}$ that is $\phi_{i} \phi_{j}=\phi_{j} \phi_{i}=0$. We say that an element $\theta_{i j}$ provides an isomorphism of degree $d$ between idempotents $\phi_{i}$ and $\phi_{j}$ if $\theta_{i j} \in \phi_{j} A^{-d} \phi_{i}$ and there exists $\theta_{j i} \in \phi_{i} A^{d} \phi_{j}$ such that $\theta_{i j} \theta_{j i}=\phi_{j}$ and $\theta_{j i} \theta_{i j}=\phi_{i}$.

2.2. ExAmple. - Let $X$ be a smooth projective irreducible variety over a field $F$ and let $\mathrm{CH}^{*}(X \times X ; \Lambda)$ be the Chow ring with coefficients in a commutative ring $\Lambda$. Set $A^{*}=$ $\operatorname{End}^{*}(\mathcal{M}(X ; \Lambda))$, where

$$
\operatorname{End}^{-i}(\mathcal{M}(X ; \Lambda))=\mathrm{CH}^{\operatorname{dim} X-i}(X \times X ; \Lambda)=\mathrm{CH}_{\operatorname{dim} X+i}(X \times X ; \Lambda), \quad i \in \mathbb{Z}
$$

and the multiplication is given by the correspondence product. By definition, $\operatorname{End}^{0}(\mathcal{M}(X ; \Lambda))$ is the ring of endomorphisms of the motive $\mathcal{M}(X ; \Lambda)$ (see 1.2). Note that a direct summand of $\mathcal{M}(X ; \Lambda)$ can be identified with a pair $\left(X, \phi_{i}\right)$, where $\phi_{i}$ is an idempotent (see [13, ch. XII]). Then an isomorphism $\theta_{i j}$ of degree $d$ between $\phi_{i}$ and $\phi_{j}$ can be identified with an isomorphism between the motives $\left(X, \phi_{i}\right)$ and $\left(X, \phi_{j}\right)(d)$.

$4^{\mathrm{e}}$ SÉRIE - TOME $41-2008-\mathrm{N}^{\mathrm{o}} 6$ 
2.3. Definition. - Let $f: A^{*} \rightarrow B^{*}$ be a homomorphism of $\mathbb{Z}$-graded rings. We say that $f$ lifts decompositions if given a family $\phi_{i} \in B^{0}$ of pair-wise orthogonal idempotents such that $\sum_{i} \phi_{i}=1_{B}$, there exists a family of pair-wise orthogonal idempotents $\varphi_{i} \in A^{0}$ such that $\sum_{i} \varphi_{i}=1_{A}$ and each $f\left(\varphi_{i}\right)$ is isomorphic to $\phi_{i}$ by means of an isomorphism of degree 0 . We say $f$ lifts decompositions strictly if, moreover, one can choose $\varphi_{i}$ such that $f\left(\varphi_{i}\right)=\phi_{i}$.

We say $f$ lifts isomorphisms if for any idempotents $\varphi_{1}$ and $\varphi_{2}$ in $A^{0}$ and any isomorphism $\theta_{12}$ of degree $d$ between idempotents $f\left(\varphi_{1}\right)$ and $f\left(\varphi_{2}\right)$ in $B^{0}$ there exists an isomorphism $\vartheta_{12}$ of degree $d$ between $\varphi_{1}$ and $\varphi_{2}$. We say $f$ lifts isomorphisms strictly if, moreover, one can choose $\vartheta_{12}$ such that $f\left(\vartheta_{12}\right)=\theta_{12}$.

2.4. - By definition we have the following properties of morphisms which lift decompositions and isomorphisms (strictly):

(i) Let $f: A^{*} \rightarrow B^{*}$ and $g: B^{*} \rightarrow C^{*}$ be two morphisms. If both $f$ and $g$ lift decompositions or isomorphisms (strictly), then so does the composite $g \circ f$.

(ii) If $g \circ f$ lifts decompositions (resp. isomorphisms) and $g$ lifts isomorphisms, then $f$ lifts decompositions (resp. isomorphisms).

(iii) Assume we are given a commutative diagram with $\operatorname{ker} f^{\prime} \subset \operatorname{im} h$

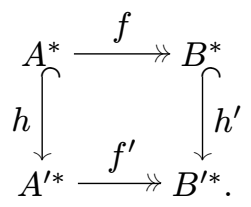

If $f^{\prime}$ lifts decompositions strictly (resp. isomorphisms strictly), then so does $f$.

2.5. Lemma. - Let $A, B$ be two rings, $A^{0}, B^{0}$ their subrings, $f^{0}: A^{0} \rightarrow B^{0}$ a ring homomorphism and $f: A \rightarrow B$ a map of sets satisfying the following conditions:

- $f(\alpha) f(\beta)$ equals either $f(\alpha \beta)$ or 0 for all $\alpha, \beta \in A$;

- $f^{0}(\alpha)$ equals $f(\alpha)$ if $f(\alpha) \in B^{0}$ or 0 otherwise;

- $\operatorname{ker} f^{0}$ consists of nilpotent elements.

Let $\varphi_{1}$ and $\varphi_{2}$ be two idempotents in $A^{0}$ and let $\psi_{12}, \psi_{21}$ be elements in $A$ such that $\psi_{12} A^{0} \psi_{21} \subset$ $A^{0}, \psi_{21} A^{0} \psi_{12} \subset A^{0}, f\left(\psi_{21}\right) f\left(\psi_{12}\right)=f\left(\varphi_{1}\right), f\left(\psi_{12}\right) f\left(\psi_{21}\right)=f\left(\varphi_{2}\right)$.

Then there exist elements $\vartheta_{12} \in \varphi_{2} A^{0} \psi_{12} A^{0} \varphi_{1}$ and $\vartheta_{21} \in \varphi_{1} A^{0} \psi_{21} A^{0} \varphi_{2}$ such that $\vartheta_{21} \vartheta_{12}=\varphi_{1}, \vartheta_{12} \vartheta_{21}=\varphi_{2}, f\left(\vartheta_{12}\right)=f\left(\varphi_{2}\right) f\left(\psi_{12}\right)=f\left(\psi_{12}\right) f\left(\varphi_{1}\right), f\left(\vartheta_{21}\right)=f\left(\varphi_{1}\right) f\left(\psi_{21}\right)=$ $f\left(\psi_{21}\right) f\left(\varphi_{2}\right)$.

Proof. - Since ker $f^{0}$ consists of nilpotents, $f^{0}$ sends non-zero idempotents in $A^{0}$ to nonzero idempotents in $B^{0}$; in particular, $f\left(\varphi_{1}\right)=f^{0}\left(\varphi_{1}\right) \neq 0, f\left(\varphi_{2}\right)=f^{0}\left(\varphi_{2}\right) \neq 0$. Observe that

$$
f\left(\psi_{12}\right) f\left(\varphi_{1}\right)=f\left(\psi_{12}\right) f\left(\psi_{21}\right) f\left(\psi_{12}\right)=f\left(\varphi_{2}\right) f\left(\psi_{12}\right)
$$

and, similarly, $f\left(\psi_{21}\right) f\left(\varphi_{2}\right)=f\left(\varphi_{1}\right) f\left(\psi_{21}\right)$. Changing $\psi_{12}$ to $\varphi_{2} \psi_{12} \varphi_{1}$ and $\psi_{21}$ to $\varphi_{1} \psi_{21} \varphi_{2}$ we may assume that $\psi_{12} \in \varphi_{2} A \varphi_{1}$ and $\psi_{21} \in \varphi_{1} A \varphi_{2}$. We have

$$
f^{0}\left(\varphi_{2}\right)=f\left(\varphi_{2}\right)=f\left(\psi_{12}\right) f\left(\psi_{21}\right)=f\left(\psi_{12} \psi_{21}\right)=f^{0}\left(\psi_{12} \psi_{21}\right)
$$


Therefore $\alpha=\psi_{12} \psi_{21}-\varphi_{2} \in A^{0}$ is nilpotent, say $\alpha^{n}=0$. Note that $\varphi_{2} \alpha=\alpha=\alpha \varphi_{2}$. Set $\alpha^{\vee}=\varphi_{2}-\alpha+\cdots+(-1)^{n-1} \alpha^{n-1} \in A^{0}$; then $\alpha \alpha^{\vee}=\varphi_{2}-\alpha^{\vee}, \varphi_{2} \alpha^{\vee}=\alpha^{\vee}=\alpha^{\vee} \varphi_{2}$ and $f\left(\varphi_{2}\right)=f^{0}\left(\varphi_{2}\right)=f^{0}\left(\alpha^{\vee}\right)=f\left(\alpha^{\vee}\right)$. Therefore setting $\vartheta_{21}=\psi_{21} \alpha^{\vee}$ we have $\vartheta_{21} \in \varphi_{1} A \varphi_{2}$,

$$
\psi_{12} \vartheta_{21}=\psi_{12} \psi_{21} \alpha^{\vee}=\left(\varphi_{2}+\alpha\right) \alpha^{\vee}=\alpha^{\vee}+\varphi_{2}-\alpha^{\vee}=\varphi_{2}
$$

and $f\left(\psi_{21}\right) f\left(\varphi_{2}\right)=f\left(\psi_{21}\right) f\left(\alpha^{\vee}\right)=f\left(\psi_{21} \alpha^{\vee}\right)=f\left(\vartheta_{21}\right)$. This also implies that $\vartheta_{21} \psi_{12}$ is an idempotent.

We have

$$
f^{0}\left(\varphi_{1}\right)=f\left(\varphi_{1}\right)=f\left(\vartheta_{21}\right) f\left(\psi_{12}\right)=f\left(\vartheta_{21} \psi_{12}\right)=f^{0}\left(\vartheta_{21} \psi_{12}\right)
$$

where the last equality holds, since $f\left(\vartheta_{21} \psi_{12}\right)=f^{0}\left(\varphi_{1}\right)$ belongs to $B^{0}$ and $f^{0}$ satisfies the second condition. Therefore $\beta=\vartheta_{21} \psi_{12}-\varphi_{1} \in A^{0}$ is nilpotent. Note that $\beta \varphi_{1}=\beta=\varphi_{1} \beta$. Now $\varphi_{1}+\beta=\left(\varphi_{1}+\beta\right)^{2}=\varphi_{1}+2 \beta+\beta^{2}$ and therefore $\beta(1+\beta)=0$. But $1+\beta$ is invertible and hence we have $\beta=0$. It means that $\vartheta_{21} \psi_{12}=\varphi_{1}$ and we can set $\vartheta_{12}=\psi_{12}$.

2.6. Proposition. - Let $f: A^{*} \rightarrow B^{*}$ be a surjective homomorphism such that the kernel of the restriction of $f$ to $A^{0}$ consists of nilpotent elements. Then $f$ lifts decompositions and isomorphisms strictly.

Proof. - The fact that $f$ lifts decompositions strictly follows from [1, Proposition 27.4].

Let $\varphi_{1}$ and $\varphi_{2}$ be two idempotents in $A^{0}$ and let $\theta_{12}$ be an isomorphism between $f\left(\varphi_{1}\right)$ and $f\left(\varphi_{2}\right)$. Let $\psi_{12} \in A$ (resp. $\psi_{21}$ ) be a homogeneous lifting of $\theta_{12}$ (resp. $\theta_{21}$ ). The proposition follows now from Lemma 2.5.

2.7. COROLlary. - Let $m$ be an integer and let $m=p_{1}^{n_{1}} \ldots p_{l}^{n_{l}}$ be its prime factorization. Then the product of reduction maps

$$
\operatorname{End}^{*}(\mathcal{M}(X ; \mathbb{Z} / m)) \rightarrow \prod_{i=1}^{l} \operatorname{End}^{*}\left(\mathcal{M}\left(X ; \mathbb{Z} / p_{i}\right)\right)
$$

lifts decompositions and isomorphisms strictly.

Proof. - We apply Proposition 2.6 to the case $A^{*}=\operatorname{End}^{*}\left(\mathcal{M}\left(X ; \mathbb{Z} / p_{i}^{n_{i}}\right)\right), B^{*}=$ $\operatorname{End}^{*}\left(\mathcal{M}\left(X ; \mathbb{Z} / p_{i}\right)\right)$ and the reduction map $f_{i}: A^{*} \rightarrow B^{*}$. We obtain that $f_{i}$ lifts decompositions and isomorphisms strictly for each $i$. To finish the proof observe that by the Chinese remainder theorem $\operatorname{End}^{*}(\mathcal{M}(X ; \mathbb{Z} / m)) \simeq \prod_{i=1}^{l} \operatorname{End}^{*}\left(\mathcal{M}\left(X ; \mathbb{Z} / p_{i}^{n_{i}}\right)\right)$.

We are coming to the following important definition.

2.8. Definition. - Let $X$ be a smooth projective variety over a field $F$. Assume that $X$ has a splitting field (see 1.4). We say that Rost Nilpotence holds for $X$ if the kernel of the restriction map

$$
\operatorname{res}_{E}: \operatorname{End}^{*}\left(\mathcal{M}\left(X_{E} ; \Lambda\right)\right) \rightarrow \operatorname{End}^{*}(\mathcal{M}(\bar{X} ; \Lambda))
$$

consists of nilpotent elements for all field extensions $E / F$ and all rings of coefficients $\Lambda$.

2.9. Lemma. - Let $X$ be a smooth projective variety which splits over any field over which it has a rational point. Then Rost Nilpotence holds for $X$.

Proof. - By [13, Theorem 67.1] if $\alpha$ is in the kernel of the restriction map $\operatorname{res}_{E}$ then $\alpha^{\circ(\operatorname{dim} X+1)}=0$.

$4^{\mathrm{e}}$ SÉRIE - TOME $41-2008-\mathrm{N}^{\mathrm{o}} 6$ 
2.10. Lemma. - Assume that Rost Nilpotence holds for $X$. Then for any field extension $E / F$ the restriction $\operatorname{res}_{E}: \operatorname{End}^{*}\left(\mathcal{M}\left(X_{E} ; \Lambda\right)\right) \rightarrow \mathrm{im}\left(\operatorname{res}_{E}\right)$ onto the image lifts decompositions and isomorphisms strictly.

Proof. - Apply Proposition 2.6 to the homomorphism $\operatorname{res}_{E}: A^{*} \rightarrow B^{*}$ between the graded rings $A^{*}=\operatorname{End}^{*}\left(\mathcal{M}\left(X_{E} ; \Lambda\right)\right)$ and $B^{*}=\operatorname{im}\left(\operatorname{res}_{E}\right)$.

2.11. Corollary. - Assume that Rost Nilpotence holds for X. Let $m$ be an integer and let $E / F$ be a field extension of degree coprime to $m$ which is rank preserving with respect to $X \times X$ (see 1.9). Then the restriction map

$$
\operatorname{res}_{E / F}: \operatorname{End}^{*}(\mathcal{M}(X ; \mathbb{Z} / m)) \rightarrow \operatorname{End}^{*}\left(\mathcal{M}\left(X_{E} ; \mathbb{Z} / m\right)\right)
$$

lifts decompositions and isomorphisms.

Proof. - By Lemma 1.10 we have im $\left(\operatorname{res}_{E}\right)=\operatorname{im}\left(\operatorname{res}_{F}\right)$. We apply now Lemma 2.10 and 2.4(ii) with $A^{*}=\operatorname{End}^{*}(\mathcal{M}(X ; \mathbb{Z} / m)), B^{*}=\operatorname{End}^{*}\left(\mathcal{M}\left(X_{E} ; \mathbb{Z} / m\right)\right)$ and $C^{*}=\operatorname{im}\left(\operatorname{res}_{E}\right)$.

2.12. Definition. - Let $V^{*}$ be a free graded $\Lambda$-module of finite rank and let $A^{*}=$ $\operatorname{End}^{*}\left(V^{*}\right)$ be its ring of endomorphisms, where $\operatorname{End}^{-d}\left(V^{*}\right), d \in \mathbb{Z}$, is the group of endomorphisms of $V^{*}$ decreasing the degree by $d$. Assume we are given a direct sum decomposition of $V^{*}=\bigoplus_{i} \operatorname{im} \phi_{i}$ by means of idempotents $\phi_{i}$ in $A^{*}$. We say that this decomposition is $\Lambda$-free if all graded components of $\operatorname{im} \phi_{i}$ are free $\Lambda$-modules. Observe that if $\Lambda=\mathbb{Z}$ or $\mathbb{Z} / p$, where $p$ is prime, then any decomposition is $\Lambda$-free.

2.13. Example. - Assume $X$ has a splitting field. Define $V^{*}=\mathrm{CH}^{*}(\bar{X})$. Then by Poincaré duality and by the Künneth decomposition (see 1.7) we have $\operatorname{End}^{*}\left(V^{*}\right)=$ $\operatorname{End}^{*}(\mathcal{M}(\bar{X}))$ (see Example 2.2).

2.14. Lemma. - The map $\mathrm{SL}_{l}(\mathbb{Z}) \rightarrow \mathrm{SL}_{l}(\mathbb{Z} / m)$ induced by the reduction modulo $m$ is surjective.

Proof. - Since $\mathbb{Z} / m$ is a semi-local ring, the group $\mathrm{SL}_{l}(\mathbb{Z} / m)$ is generated by elementary matrices (see [18, Theorem 4.3.9]).

2.15. Proposition. - Consider a free graded $\mathbb{Z}$-module $V^{*}$ of finite rank and the reduction map $f: \operatorname{End}^{*}\left(V^{*}\right) \rightarrow \operatorname{End}^{*}\left(V^{*} \otimes_{\mathbb{Z}} \mathbb{Z} / m\right)$. Then $f$ lifts $\mathbb{Z} / m$-free decompositions strictly. Moreover, if $(\mathbb{Z} / m)^{\times}=\{ \pm 1\}$, then $f$ lifts isomorphisms of $\mathbb{Z} / m$-free decompositions strictly.

Proof. - We are given a decomposition $V^{k} \otimes_{\mathbb{Z}} \mathbb{Z} / m=\oplus_{i} W_{i}^{k}$, where $W_{i}^{k}$ is the $k$-graded component of $\operatorname{im} \phi_{i}$. Present $V^{k}$ as a direct sum $V^{k}=\bigoplus_{i} V_{i}^{k}$ of free $\mathbb{Z}$-modules such that $\mathrm{rk}_{\mathbb{Z}} V_{i}^{k}=\mathrm{rk}_{\mathbb{Z} / m} W_{i}^{k}$. Fix a $\mathbb{Z}$-basis $\left\{v_{i j}^{k}\right\}_{j}$ of $V_{i}^{k}$. For each $W_{i}^{k}$ choose a basis $\left\{w_{i j}^{k}\right\}_{j}$ such that the linear transformation $D^{k}$ of $V^{k} \otimes_{\mathbb{Z}} \mathbb{Z} / m$ sending each $v_{i j}^{k} \otimes 1$ to $w_{i j}^{k}$ has determinant 1 . By Lemma 2.14 there is a lifting $\tilde{D}^{k}$ of $D^{k}$ to a linear transformation of $V^{k}$. So we obtain $V^{k}=\bigoplus_{i} \tilde{W}_{i}^{k}$, where $\tilde{W}_{i}^{k}=\tilde{D}^{k}\left(V_{i}^{k}\right)$ satisfies $\tilde{W}_{i}^{k} \otimes_{\mathbb{Z}} \mathbb{Z} / m=W_{i}^{k}$. It remains to define $\varphi_{i}$ on each $V^{k}$ to be the projection onto $\tilde{W}_{i}^{k}$.

Now let $\varphi_{1}, \varphi_{2}$ be two idempotents in $\operatorname{End}^{*}\left(V^{*}\right)$. Let $V_{i}^{k}$ denote the $k$-graded component of $\operatorname{im} \varphi_{i}$. An isomorphism $\theta_{12}$ between $\varphi_{1} \otimes 1$ and $\varphi_{2} \otimes 1$ of degree $d$ can be identified with a family of isomorphisms $\theta_{12}^{k}: V_{1}^{k} \otimes \mathbb{Z} / m \rightarrow V_{2}^{k-d} \otimes \mathbb{Z} / m$. In the case $(\mathbb{Z} / m)^{\times}=\{ \pm 1\}$ all 
these isomorphisms are given by matrices with determinants $\{ \pm 1\}$ and, hence, can be lifted to isomorphisms $\vartheta_{12}^{k}: V_{1}^{k} \rightarrow V_{2}^{k-d}$ by Lemma 2.14.

Now we are ready to state and to prove the main result of this section.

2.16. Theorem. - Let $X$ be a smooth projective irreducible variety over a field $F$. Assume that $X$ has a splitting field of degree $m$ which is rank preserving with respect to $X \times X$. Assume that Rost Nilpotence holds for $X$. Consider only decompositions of $\mathcal{M}(X ; \mathbb{Z} / m)$ which become $\mathbb{Z} / m$-free over the splitting field. Then the reduction map

$$
f: \operatorname{End}^{*}(\mathcal{M}(X ; \mathbb{Z})) \rightarrow \operatorname{End}^{*}(\mathcal{M}(X ; \mathbb{Z} / m))
$$

lifts such decompositions. If additionally $(\mathbb{Z} / m)^{\times}=\{ \pm 1\}$, then this map lifts isomorphisms of such decompositions.

Proof. - Consider the diagram

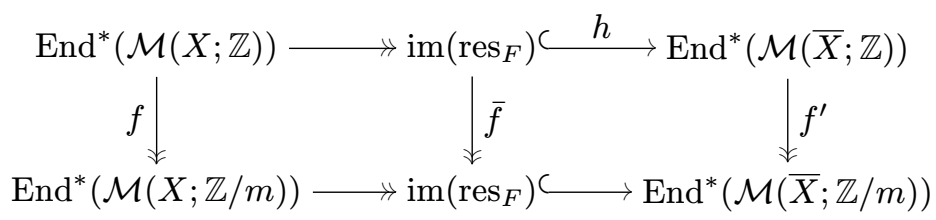

Recall that we can identify End ${ }^{-d}(\mathcal{M}(\bar{X}))$ with the group of endomorphisms of $\mathrm{CH}^{*}(\bar{X})$ which decrease the grading by $d$ (see 2.13). Applying Proposition 2.15 to the case $V^{*}=$ $\mathrm{CH}^{*}(\bar{X})$ we obtain that the map $f^{\prime}$ lifts decompositions strictly. Moreover, if $(\mathbb{Z} / m)^{\times}=$ $\{ \pm 1\}$ then $f^{\prime}$ lifts isomorphisms strictly.

By Lemma $1.10 \operatorname{ker} f^{\prime} \subset \operatorname{im} h$ and, therefore, applying 2.4(iii) we obtain that $\bar{f}$ lifts decompositions strictly and, moreover, $\bar{f}$ lifts isomorphisms strictly if $(\mathbb{Z} / m)^{\times}=\{ \pm 1\}$.

Now by Lemma 2.10 the horizontal arrows of the left square lift decompositions and isomorphisms strictly. It remains to apply 2.4(i) and (ii).

\section{Motives of fibered spaces}

In the present section we discuss motives of cellular fibration. The main result (Theorem 3.7) generalizes and uniformizes the proofs of paper [5].

3.1. Definition. - Let $X$ be a smooth projective variety over a field $F$. We say a smooth projective morphism $f: Y \rightarrow X$ is a cellular fibration if it is a locally trivial fibration whose fiber $\mathcal{F}$ is cellular, i.e. has a decomposition into affine cells (see $[13, \S 66]$ ).

3.2. Lemma. - Let $f: Y \rightarrow X$ be a cellular fibration. Then $\mathcal{M}(Y)$ is isomorphic to $\mathcal{M}(X) \otimes \mathcal{M}(\mathcal{F})$

$4^{\text {e }}$ SÉRIE - TOME $41-2008-\mathrm{N}^{\mathrm{o}} 6$ 
Proof. - We follow the proof of [12, Proposition 1]. Define the morphism

$$
\varphi: \bigoplus_{i \in \mathcal{I}} \mathcal{M}(X)\left(\operatorname{codim} B_{i}\right) \rightarrow \mathcal{M}(Y)
$$

to be the direct sum $\varphi=\bigoplus_{i \in \mathcal{I}} \varphi_{i}$, where each $\varphi_{i}$ is given by the cycle $\left[\operatorname{pr}_{Y}^{*}\left(B_{i}\right) \cdot \Gamma_{f}\right] \in$ $\mathrm{CH}(X \times Y)$ produced from the graph cycle $\Gamma_{f}$ and the chosen (non-canonical) basis $\left\{B_{i}\right\}_{i \in \mathcal{I}}$ of $\mathrm{CH}(Y)$ over $\mathrm{CH}(X)$. The realization of $\varphi$ coincides exactly with the isomorphism of abelian groups $\mathrm{CH}(X) \otimes \mathrm{CH}(\mathcal{F}) \rightarrow \mathrm{CH}(Y)$ constructed in [12, Proposition 1]. Then by Manin's identity principle (see $[26, \S 3]) \varphi$ is an isomorphism.

3.3. Lemma. - Let $G$ be a linear algebraic group over a field $F$ and let $X$ be a projective homogeneous $G$-variety. Let $Y$ be a $G$-variety and let $f: Y \rightarrow X$ be a $G$-equivariant projective morphism. Assume that the fiber of $f$ over $F(X)$ is isomorphic to $\mathcal{F}_{F(X)}$ for some variety $\mathcal{F}$ over $F$. Then $f$ is a locally trivial fibration with fiber $\mathcal{F}$.

Proof. - By the assumptions, we have $Y \times_{X} \operatorname{Spec} F(X) \simeq(\mathcal{F} \times X) \times{ }_{X} \operatorname{Spec} F(X)$ as schemes over $F(X)$. Since $F(X)$ is a direct limit of $\mathcal{O}(U)$ taken over all non-empty affine open subsets $U$ of $X$, by [EGA IV, Corollaire 8.8.2.5] there exists $U$ such that $f^{-1}(U)=$ $Y \times{ }_{X} U$ is isomorphic to $(\mathcal{F} \times X) \times{ }_{X} U \simeq \mathcal{F} \times U$ as a scheme over $U$. Since $G$ acts transitively on $X$ and $f$ is $G$-equivariant, the map $f$ is a locally trivial fibration.

3.4. Corollary. - Let $X$ be a projective homogeneous $G$-variety and let $Y$ be a projective variety such that $Y_{F(X)} \simeq \mathcal{F}_{F(X)}$ for some variety $\mathcal{F}$. Then the projection map $X \times Y \rightarrow X$ is a locally trivial fibration with fiber $\mathcal{F}$. Moreover, if $\mathcal{F}$ is cellular, then $\mathcal{M}(X \times Y) \simeq \mathcal{M}(X) \otimes$ $\mathcal{M}(\mathcal{F})$.

Proof. - Apply Lemma 3.3 to the projection map $X \times Y \rightarrow X$ and use Lemma 3.2.

3.5. Lemma. - Let $G$ be a semisimple linear algebraic group over $F$. Let $X$ and $Y$ be projective homogeneous $G$-varieties corresponding to parabolic subgroups $P$ and $Q$ of the split form $G_{0}, Q \subseteq P$. Let $f: Y \rightarrow X$ be the map induced by the quotient map. If $G$ splits over $F(X)$ then $f$ is a cellular fibration with fiber $\mathcal{F}=P / Q$.

Proof. - Since $G$ splits over $F(X)$, the fiber of $f$ over $F(X)$ is isomorphic to $(P / Q)_{F(X)}=$ $\mathcal{F}_{F(X)}$. Now apply Lemma 3.3 and note that $\mathcal{F}$ is cellular.

3.6. EXAMPLE. - Let $P=P_{\Theta}$ be the standard parabolic subgroup of a split simple group $G_{0}$ corresponding to a subset $\Theta$ of the respective Dynkin diagram $\mathcal{D}$ (our enumeration of roots follows Bourbaki). In this notation the Borel subgroup corresponds to the empty set. Let $\xi$ be a cocycle in $Z^{1}\left(F, G_{0}\right)$. Set $G={ }_{\xi} G_{0}$ and $X={ }_{\xi}\left(G_{0} / P\right)$. In particular, $G$ is a group of inner type and $X$ is the respective projective homogeneous $G$-variety. Let $q$ denote the degree of a splitting field of $G$ and let $d$ be the index of the associated Tits algebra (see [34, Table II]). For groups of type $\mathrm{D}_{n}$, we set $d$ to be the index of the Tits algebra associated with the vector representation.

Analyzing Tits indices of $G$ we see that $G$ becomes split over $F(X)$ if the subset $\mathcal{D} \backslash \Theta$ contains one of the following vertices $k$ (cf. $[23, \S 7]$ ): 


\begin{tabular}{l||l|l|l|l|l}
$G_{0}$ & ${ }^{1} \mathrm{An}_{\mathrm{n}}$ & $\mathrm{B}_{\mathrm{n}}$ & $\mathrm{C}_{\mathrm{n}}$ & ${ }^{1} \mathrm{D}_{\mathrm{n}}$ & $\mathrm{G}_{2}$ \\
\hline$k$ & $\operatorname{gcd}(k, d)=1$ & $\begin{array}{l}k=n ; \\
\text { any } k \text { in the } \\
\text { Pfister case }\end{array}$ & $k$ is odd; & $\begin{array}{l}k=n-1, n \text { if } d=1 ; \\
\text { any } k \text { in the } \\
\text { Pfister case }\end{array}$ & any \\
\end{tabular}

\begin{tabular}{l||l|l|l|l}
$G_{0}$ & $\mathrm{~F}_{4}$ & ${ }^{1} \mathrm{E}_{6}$ & $\mathrm{E}_{7}$ & $\mathrm{E}_{8}$ \\
\hline$k$ & $k=1,2,3 ;$ & $k=3,5 ;$ & $k=2,5 ;$ & $k=2,3,4,5 ;$ \\
& any $k$ if & $k=2,4$ if $d=1 ;$ & $k=3,4$ if $d=1 ;$ & any $k$ if \\
& $q=3$ & $k=1,6$ if $q$ is odd & $k \neq 7$ if $q=3$ & $q=5$
\end{tabular}

(here by the Pfister case we mean the case when the cocycle $\xi$ corresponds to a Pfister form or its maximal neighbor).

Case-by-case arguments from the paper [5] show that under certain conditions the Chow motive of a twisted flag variety $X$ can be expressed in terms of the motive of a minimal flag. These conditions cover almost all twisted flag varieties corresponding to groups of types $\mathrm{A}_{n}$ and $\mathrm{B}_{n}$ together with some examples of types $\mathrm{C}_{n}, \mathrm{G}_{2}$ and $\mathrm{F}_{4}$. The following theorem together with Table 3.6 provides a uniform proof of these results and extends them to some other types.

3.7. Theorem. - Let $Y$ and $X$ be taken as in Lemma 3.5. Then the Chow motive $\mathcal{M}(Y)$ of $Y$ is isomorphic to a direct sum of twisted copies of the motive $\mathcal{M}(X)$, i.e.

$$
\mathcal{M}(Y) \simeq \bigoplus_{i \geq 0} \mathcal{M}(X)(i)^{\oplus c_{i}}
$$

where $\sum c_{i} t^{i}=P\left(\mathrm{CH}_{*}(\bar{Y}), t\right) / P\left(\mathrm{CH}_{*}(\bar{X}), t\right)$ is the quotient of the respective Poincaré polynomials.

Proof. - Apply Lemmas 3.5 and 3.2.

3.8. Remark. - An explicit formula for $P\left(\mathrm{CH}_{*}(\bar{X}), t\right)$ involves degrees of the basic polynomial invariants of $G_{0}$ and is provided in [19, Ch. IV, Cor. 4.5].

\section{4. $J$-invariant and its properties}

Fix a prime integer $p$. To simplify the notation we denote the Chow ring of a variety $X$ with $\mathbb{Z} / p$-coefficients by $\mathrm{Ch}(X)$ and the image of the restriction map $\mathrm{CH}(X ; \mathbb{Z} / p) \rightarrow \mathrm{CH}(\bar{X} ; \mathbb{Z} / p)$ by $\overline{\mathrm{Ch}}(X)$.

4.1. - Let $G_{0}$ be a split semisimple linear algebraic group over a field $F$ with a split maximal torus $T$ and a Borel subgroup $B$ containing $T$. Let $G={ }_{\xi} G_{0}$ be a twisted form of $G_{0}$ given by a cocycle $\xi \in Z^{1}\left(F, G_{0}\right)$. Let $\mathfrak{X}={ }_{\xi}\left(G_{0} / B\right)$ be the corresponding variety of complete flags. Observe that the group $G$ splits over any field $K$ over which $\mathfrak{X}$ has a rational point, in particular, over the function field $F(\mathfrak{X})$. According to [10] the Chow $\operatorname{ring} \mathrm{Ch}(\overline{\mathfrak{X}})$ can be expressed in purely combinatorial terms and, therefore, depends only on the type of $G$ but not on the base field $F$. 
4.2. - Let $\hat{T}$ be the group of characters of $T$ and let $S(\hat{T})$ be the symmetric algebra. We denote by $R$ the image of the characteristic map $c: S(\hat{T}) \rightarrow \mathrm{Ch}(\overline{\mathfrak{X}})$ (see [17, (4.1)]). According to [22, Thm.6.4] there is an embedding

$$
R \subseteq \overline{\mathrm{Ch}}(\mathfrak{X}),
$$

where the equality holds if the cocycle $\xi$ corresponds to a generic torsor.

4.3. - Let $\mathrm{Ch}(\bar{G})$ denote the Chow ring with $\mathbb{Z} / p$-coefficients of the group $G_{0}$ over a splitting field of $\overline{\mathfrak{X}}$. Consider the pull-back induced by the quotient map

$$
\pi: \operatorname{Ch}(\overline{\mathfrak{X}}) \rightarrow \operatorname{Ch}(\bar{G}) .
$$

According to $\left[17\right.$, p. 21 , Rem. $\left.2^{\circ}\right] \pi$ is surjective with its kernel generated by $R^{+}$, where $R^{+}$ stands for the subgroup of the non-constant elements of $R$.

4.4. - An explicit presentation of $\operatorname{Ch}(\bar{G})$ is known for all types of $G$ and all torsion primes $p$ of $G$ (in the sense of [9, Proposition 6.(a)]) provided in Table 4.13. Namely, by [20, Theorem 3] it is a quotient of the polynomial ring in $r$ variables $x_{1}, \ldots, x_{r}$ of codimensions $d_{1} \leq d_{2} \leq \cdots \leq d_{r}$ coprime to $p$, modulo an ideal generated by certain $p$-powers $x_{1}^{p^{k_{1}}}, \ldots, x_{r}^{p^{k_{r}}}\left(k_{i} \geq 0, i=1, \ldots, r\right)$

$$
\mathrm{Ch}^{*}(\bar{G})=(\mathbb{Z} / p)\left[x_{1}, \ldots, x_{r}\right] /\left(x_{1}^{p^{k_{1}}}, \ldots, x_{r}^{p^{k_{r}}}\right) .
$$

In the case where $p$ is not a torsion prime of $G$ we have $\mathrm{Ch}^{*}(\bar{G})=\mathbb{Z} / p$, i.e. $r=0$.

Note that a complete list of numbers $\left\{d_{i} p^{k_{i}}\right\}_{i=1 \ldots r}$ called $p$-exceptional degrees of $G_{0}$ is provided in [20, Table II]. Taking the $p$-primary and $p$-coprimary parts of each $p$-exceptional degree from this table one restores the respective $k_{i}$ and $d_{i}$.

4.5. Definition. - We introduce two orders on the set of additive generators of $\mathrm{Ch}(\bar{G})$, i.e. on the monomials $x_{1}^{m_{1}} \ldots x_{r}^{m_{r}}$. To simplify the notation, we will denote the monomial $x_{1}^{m_{1}} \ldots x_{r}^{m_{r}}$ by $x^{M}$, where $M$ is an $r$-tuple of integers $\left(m_{1}, \ldots, m_{r}\right)$. The codimension of $x^{M}$ will be denoted by $|M|$. Observe that $|M|=\sum_{i=1}^{r} d_{i} m_{i}$.

- Given two $r$-tuples $M=\left(m_{1}, \ldots, m_{r}\right)$ and $N=\left(n_{1}, \ldots, n_{r}\right)$ we say $x^{M} \preccurlyeq x^{N}$ (or equivalently $M \preccurlyeq N$ ) if $m_{i} \leq n_{i}$ for all $i$. This gives a partial ordering on the set of all monomials ( $r$-tuples).

- Given two $r$-tuples $M=\left(m_{1}, \ldots, m_{r}\right)$ and $N=\left(n_{1}, \ldots, n_{r}\right)$ we say $x^{M} \leq x^{N}$ (or equivalently $M \leq N)$ if either $|M|<|N|$, or $|M|=|N|$ and $m_{i} \leq n_{i}$ for the greatest $i$ such that $m_{i} \neq n_{i}$. This gives a well-ordering on the set of all monomials ( $r$-tuples) known as the DegLex order.

Now we are ready to give the main definition of the present paper.

4.6. Definition. - Let $G={ }_{\xi} G_{0}$ be the twisted form of a split semisimple algebraic group $G_{0}$ over a field $F$ by means of a cocycle $\xi \in Z^{1}\left(F, G_{0}\right)$ and let $\mathfrak{X}={ }_{\xi}\left(G_{0} / B\right)$ be the respective variety of complete flags. Let $\mathfrak{C h}(G)$ denote the image of the composite

$$
\mathrm{Ch}(\mathfrak{X}) \stackrel{\text { res }}{\longrightarrow} \mathrm{Ch}(\overline{\mathfrak{X}}) \stackrel{\pi}{\rightarrow} \mathrm{Ch}(\bar{G}) .
$$

Since both maps are ring homomorphisms, $\mathfrak{C h}(G)$ is a subring of $\mathrm{Ch}(\bar{G})$. 
For each $1 \leq i \leq r$ set $j_{i}$ to be the smallest non-negative integer such that the subring $\mathfrak{C h}(G)$ contains an element $a$ with the greatest monomial $x_{i}^{p^{j_{i}}}$ with respect to the DegLex order on $\operatorname{Ch}(\bar{G})$, i.e. of the form

$$
a=x_{i}^{p^{j_{i}}}+\sum_{x^{M} \lesseqgtr x_{i}^{p_{i}}} c_{M} x^{M}, \quad c_{M} \in \mathbb{Z} / p .
$$

The $r$-tuple of integers $\left(j_{1}, \ldots, j_{r}\right)$ will be called the $J$-invariant of $G$ modulo $p$ and will be denoted by $J_{p}(G)$.

Observe that if the Chow ring $\operatorname{Ch}(\bar{G})$ has only one generator, i.e. $r=1$, then the $J$-invariant is equal to the smallest non-negative integer $j_{1}$ such that $x_{1}^{p^{j_{1}}} \in \mathfrak{C h}(G)$.

4.7. Example. - By definition it follows that $J_{p}\left(G_{E}\right) \preccurlyeq J_{p}(G)$ for any field extension $E / F$. Moreover, $J_{p}(G) \preccurlyeq\left(k_{1}, \ldots, k_{r}\right)$ by $(2)$.

According to (1) the $J$-invariant takes its maximal possible value $J_{p}(G)=\left(k_{1}, \ldots, k_{r}\right)$ if the cocycle $\xi$ corresponds to a generic torsor. Later on (see Corollary 6.7) it will be shown that the $J$-invariant takes its minimal value $J_{p}(G)=(0, \ldots, 0)$ if and only if the group $G$ splits by a finite field extension of degree coprime to $p$.

The next example explains the terminology ' $J$-invariant'.

4.8. Example. - Let $\phi$ be a quadratic form with trivial discriminant. In [35, Definition 5.11] A. Vishik introduced the notion of the $J$-invariant of $\phi$, a set of integers which describes the subgroup of rational cycles on the respective maximal orthogonal Grassmannian. This invariant provides an important tool for the study of algebraic cycles on quadrics. In particular, it was one of the main ingredients used by A. Vishik in his significant progress on the solution of Kaplansky's Problem. More precisely, in the notation of paper [36] the $J$-invariant of a quadric corresponds to the upper row of its elementary discrete invariant (see [36, Definition 2.2]).

An equivalent but slightly different definition of the $J$-invariant of $\phi$ can be found in [13, $\S 88]$. Namely, if $J(\phi)$ denotes the $J$-invariant of $\phi$ from [13], then Vishik's $J$-invariant is equal to $\{0,1, \ldots, n\} \backslash J(\phi)$ in the case $\operatorname{dim} \phi=2 n$ and $\{1, \ldots, n\} \backslash J(\phi)$ in the case $\operatorname{dim} \phi=2 n-1$.

Using Theorem 3.7 one can show that $J(\phi)$ introduced in [13] can be expressed in terms of $J_{2}\left(\mathrm{O}^{+}(\phi)\right)=\left(j_{1}, \ldots, j_{r}\right)$ as follows:

$$
J(\phi)=\left\{2^{l} d_{i} \mid i=1, \ldots, r, 0 \leq l \leq j_{i}-1\right\} .
$$

Since all $d_{i}$ are odd, $J_{2}\left(\mathrm{O}^{+}(\phi)\right)$ is uniquely determined by $J(\phi)$.

We have the following reduction formula (cf. [13, Cor. 88.6] in the case of quadrics).

4.9. Proposition. - Let $G$ be a semisimple group of inner type over a field $F$ and let $\mathfrak{X}$ be the variety of complete $G$-flags. Let $Y$ be a projective variety such that the map $\operatorname{Ch}^{l}(Y) \rightarrow$ $\mathrm{Ch}^{l}\left(Y_{F(x)}\right)$ is surjective for all $x \in X$ and $l \leq n$. Then $j_{i}(G)=j_{i}\left(G_{F(Y)}\right)$ for all $i$ such that $d_{i} p^{j_{i}\left(G_{F(Y)}\right)} \leq n$.

Proof. - By [13, Lemma 88.5] the map $\mathrm{Ch}^{l}(X) \rightarrow \mathrm{Ch}^{l}\left(X_{F(Y)}\right)$ is surjective for all $l \leq n$ and, therefore $j_{i}(G) \leq j_{i}\left(G_{F(Y)}\right)$. The converse inequality is obvious. 


\subsection{Corollary. $-J_{p}(G)=J_{p}\left(G_{F(t)}\right)$.}

Proof. - Take $Y=\mathbb{P}^{1}$ and apply Proposition 4.9.

4.11. - To find restrictions on the possible values of $J_{p}(G)$ we use Steenrod $p$-th power operations introduced by P. Brosnan. Recall (see [3]) that if the characteristic of the base field $F$ is different from $p$ then one can construct Steenrod $p$-th power operations

$$
S^{l}: \mathrm{Ch}^{*}(X) \rightarrow \mathrm{Ch}^{*+l(p-1)}(X), \quad l \geq 0
$$

such that $S^{0}=\mathrm{id}$, the restriction $\left.S^{l}\right|_{\mathrm{Ch}^{l}(X)}$ coincides with taking to the $p$-th power, $\left.S^{l}\right|_{\mathrm{Ch}^{i}(X)}=0$ for $l>i$, and the total operation $S^{\bullet}=\sum_{l \geq 0} S^{l}$ is a homomorphism of $\mathbb{Z} / p$ algebras compatible with pull-backs. In particular, Steenrod operations preserve rationality of cycles.

In the case of projective homogeneous varieties over the field of complex numbers $S^{l}$ is compatible with its topological counterparts: the reduced power operation $\mathcal{P}^{l}$ if $p \neq 2$ and the Steenrod square $S q^{2 l}$ if $p=2$ (over complex numbers $\mathrm{Ch}^{*}(\overline{\mathfrak{X}})$ can be viewed as a subring of the singular cohomology $\left.\mathrm{H}_{\text {sing }}^{2 *}(\overline{\mathfrak{X}}, \mathbb{Z} / p)\right)$. Moreover, $\mathrm{Ch}^{*}(\bar{G})$ may be identified with the image of the pull-back map $\mathrm{H}_{\text {sing }}^{2 *}(\overline{\mathfrak{X}}, \mathbb{Z} / p) \rightarrow \mathrm{H}_{\text {sing }}^{2 *}(\bar{G}, \mathbb{Z} / p)$. An explicit description of this image and formulae describing the action of $\mathcal{P}^{l}$ and $S q^{2 l}$ on $\mathrm{H}_{\text {sing }}^{*}(\bar{G}, \mathbb{Z} / p)$ can be found in [29] for exceptional groups and in [13] for classical groups.

The action of the Steenrod operations on $\mathrm{Ch}(\overline{\mathfrak{X}})$ and on $\mathrm{Ch}(\bar{G})$ can be described in purely combinatorial terms (see [11]) and, hence, does not depend on the choice of a base field $F$.

The following lemma provides an important technical tool for computing possible values of the $J$-invariant of $G$.

4.12. Lemma. - Assume that in $\mathrm{Ch}^{*}(\bar{G})$ we have $S^{l}\left(x_{i}\right)=x_{m}^{p^{s}}$, and for any $i^{\prime}<i$ $S^{l}\left(x_{i^{\prime}}\right)<x_{m}^{p^{s}}$ with respect to the DegLex order. Then $j_{m} \leq j_{i}+s$.

Proof. - By definition there exists a cycle $\alpha \in \overline{\mathrm{Ch}}(\mathfrak{X})$ such that the leading term of $\pi(\alpha)$ is $x_{i}^{p^{j_{i}}}$. For the total operation we have

$$
S\left(x_{i}^{p^{j_{i}}}\right)=S\left(x_{i}\right)^{p^{j_{i}}}=S^{0}\left(x_{i}\right)^{p^{j_{i}}}+S^{1}\left(x_{i}\right)^{p^{j_{i}}}+\cdots+S^{d_{i}}\left(x_{i}\right)^{p^{j_{i}}} .
$$

In particular, $S^{l p^{j_{i}}}\left(x_{i}^{p^{j_{i}}}\right)=S^{l}\left(x_{i}\right)^{p^{j_{i}}}$. Applying $S^{l p^{j_{i}}}$ to $\alpha$ we obtain a rational cycle whose image under $\pi$ has the leading term $x_{m}^{p^{j_{i}+s}}$.

4.13. - We summarize information about restrictions on the $J$-invariant into the following table (numbers $r, d_{i}$ and $k_{i}$ are taken from [20, Table II]). For groups we use the notation from [24].

Recall that $r$ is the number of generators of $\mathrm{Ch}^{*}(\bar{G}), d_{i}$ are their codimensions, $k_{i}$ define the $p$-power relations, $J_{p}(G)=\left(j_{1}, \ldots, j_{r}\right), 0 \leq j_{i} \leq k_{i}$ for all $i$, and $G={ }_{\xi}\left(G_{0}\right)$ for some $\xi \in H^{1}\left(F, G_{0}\right)$. If $p$ is not in the table, then $J_{p}(G)=()$ is empty. 


\begin{tabular}{|c|c|c|c|c|c|}
\hline$G_{0}$ & $p$ & $r$ & $k_{i}, i=1 \ldots r$ & $d_{i}, i=1 \ldots r$ & restrictions on $j i$ \\
\hline $\mathrm{SLn} / \mu m, m \mid n$ & $p \mid m$ & 1 & $p^{k_{1}} \| n$ & 1 & \\
\hline $\mathrm{PGSp}_{\mathrm{n}}, 2 \mid n$ & 2 & 1 & $2^{1} \| n$ & 1 & \\
\hline $\mathrm{O}^{+}, n>2$ & 2 & {$\left[\frac{\mathrm{n}+1}{4}\right]$} & {$\left[\log _{2} \frac{-1}{2-1}\right]$} & $2 i-1$ & $\begin{array}{l}j i \geq j i_{+} \text {if } 2 \nmid\left({ }^{-1}\right), \\
j i \leq j_{2}-1+1\end{array}$ \\
\hline $\operatorname{Spin}_{\mathrm{n}}, n>2$ & 2 & {$\left[\frac{\mathrm{n}-3}{4}\right]$} & {$\left[\log _{2} \frac{-1}{2+1}\right]$} & $2 i+1$ & $\begin{array}{l}j i \geq j i_{+} \text {if } 2 \nmid() \\
j i \leq j_{2}+1\end{array}$ \\
\hline $\mathrm{PGO}_{2}^{+}, n>1$ & 2 & {$\left[\frac{\mathrm{n}+2}{2}\right]$} & $\begin{array}{l}2^{1} \| n \\
{\left[\log _{2} \frac{2}{2-3}\right]}\end{array}$ & $\begin{array}{l}1, i=1 \\
2 i-3, i \geq 2\end{array}$ & $\begin{array}{l}j i \geq j i_{+} \text {if } 2 \nmid\left({ }^{-2}\right), \\
j i \leq j_{2}-2+1\end{array}$ \\
\hline $\operatorname{Spin}_{2}^{ \pm}, 2 \mid n$ & 2 & $\frac{n}{2}$ & $\begin{array}{l}2^{1}|| n \\
{\left[\log _{2} \frac{2}{2-1}\right]}\end{array}$ & $\begin{array}{l}1, i=1 \\
2 i-1, i \geq 2\end{array}$ & $\begin{array}{l}j i \geq j i_{+} \text {if } 2 \nmid\left({ }^{-1}\right) \\
j i \leq j_{2}+1\end{array}$ \\
\hline $\mathrm{G}_{2}, \mathrm{~F}_{4}, \mathrm{E}_{6}$ & 2 & 1 & 1 & 3 & \\
\hline $\mathrm{F}_{4}, \mathrm{E}_{6}^{\mathrm{sc}}, \mathrm{E}_{7}$ & 3 & 1 & 1 & 4 & \\
\hline $\mathrm{E}_{6}^{\mathrm{ad}}$ & 3 & 2 & 2,1 & 1,4 & \\
\hline $\mathrm{E}_{7}^{\mathrm{sc}}$ & 2 & 3 & $1,1,1$ & $3,5,9$ & $j_{1} \geq j_{2} \geq j_{3}$ \\
\hline $\mathrm{E}_{7}^{\mathrm{ad}}$ & 2 & 4 & $1,1,1,1$ & $1,3,5,9$ & $j_{2} \geq j_{3} \geq j_{4}$ \\
\hline $\mathrm{E}_{8}$ & 2 & 4 & $3,2,1,1$ & $3,5,9,15$ & $\begin{array}{l}j_{1} \geq j_{2} \geq j_{3} \\
j_{1} \leq j_{2}+1 \\
j_{2} \leq j_{3}+1\end{array}$ \\
\hline $\mathrm{E}_{8}$ & 3 & 2 & 1,1 & 4,10 & $j_{1} \geq j_{2}$ \\
\hline $\mathrm{E}_{8}$ & 5 & 1 & 1 & 6 & \\
\hline
\end{tabular}

The upper indices ${ }^{\mathrm{sc}}$ and ${ }^{\text {ad }}$ denote simply-connected and adjoint groups respectively. The last column of the table follows from Lemma 4.12 and, hence, requires char $(F) \neq p$ restriction. All other columns are taken directly from [20, Table II] and are independent on the characteristic of the base field.

\section{Motivic decompositions}

In the present section we prove the main result of this paper (Theorem 5.17). First, we describe a basis of the subring of rational cycles of $\mathrm{Ch}(\overline{\mathfrak{X}} \times \overline{\mathfrak{X}})$, where $\mathfrak{X}$ is the variety of complete flags. The key results here are Propositions 5.3 and 5.10. As a consequence, we obtain a motivic decomposition of $\mathfrak{X}$ (Theorem 5.13) in terms of a certain motive $\mathcal{R}_{p}(G)$. Then using motivic decompositions of cellular fibrations (see Theorem 3.7) we generalize Theorem 5.13 to arbitrary generically split projective homogeneous varieties. At the end we discuss some properties of the motive $\mathcal{R}_{p}(G)$.

5.1. - We use the notation of the previous section. Let $G$ be a semi-simple group of inner type over $F$ and let $\mathfrak{X}$ be the respective variety of complete flags. Let $R \subseteq \overline{\mathrm{Ch}}(\mathfrak{X})$ be the image of the characteristic map. Consider the quotient map $\pi: \operatorname{Ch}(\overline{\mathfrak{X}}) \rightarrow \operatorname{Ch}(\bar{G})$. Fix preimages $e_{i}$ of $x_{i}$ in $\operatorname{Ch}(\overline{\mathfrak{X}})$. For an $r$-tuple $M=\left(m_{1}, \ldots, m_{r}\right)$ set $e^{M}=\prod_{i=1}^{r} e_{i}^{m_{i}}$. Set $N=\left(p^{k_{1}}-\right.$ $\left.1, \ldots, p^{k_{r}}-1\right)$ and $d=\operatorname{dim} \mathfrak{X}-|N|$.

5.2. Lemma. - The Chow ring $\mathrm{Ch}(\overline{\mathfrak{X}})$ is a free $R$-module with a basis $\left\{e^{M}\right\}, M \preccurlyeq N$. 
Proof. - Note that the subgroup $R^{+}$of the non-constant elements of $R$ is a nilpotent ideal in $R$. Applying the Nakayama Lemma we obtain that $\left\{e^{M}\right\}$ generates $\operatorname{Ch}(\overline{\mathfrak{X}})$. By $[20$, (2)] $\mathrm{Ch}(\overline{\mathfrak{X}})$ is a free $R$-module, hence, for the Poincaré polynomials we have

$$
P\left(\mathrm{Ch}^{*}(\overline{\mathfrak{X}}), t\right)=P\left(\mathrm{Ch}^{*}(\bar{G}), t\right) \cdot P\left(R^{*}, t\right) .
$$

Substituting $t=1$ we obtain that

$$
\operatorname{rk} \operatorname{Ch}(\overline{\mathfrak{X}})=\operatorname{rk} \operatorname{Ch}(\bar{G}) \cdot \operatorname{rk} R .
$$

To finish the proof observe that $\operatorname{rk} \operatorname{Ch}(\bar{G})$ coincides with the number of generators $\left\{e^{M}\right\}$.

5.3. Proposition. - The pairing $R \times R \rightarrow \mathbb{Z} / p$ given by $(\alpha, \beta) \mapsto \operatorname{deg}\left(e^{N} \alpha \beta\right)$ is nondegenerated, i.e. for any non-zero element $\alpha \in R$ there exists $\beta$ such that $\operatorname{deg}\left(e^{N} \alpha \beta\right) \neq 0$.

Proof. - Choose a homogeneous basis of $\mathrm{Ch}(\overline{\mathfrak{X}})$. Let $\alpha^{\vee}$ be the Poincaré dual of $\alpha$ with respect to this basis. By Lemma $5.2 \mathrm{Ch}(\overline{\mathfrak{X}})$ is a free $R$-module with the basis $\left\{e^{M}\right\}$, hence, expanding $\alpha^{\vee}$ we obtain

$$
\alpha^{\vee}=\sum_{M \preccurlyeq N} e^{M} \beta_{M}, \text { where } \beta_{M} \in R .
$$

Note that if $M \neq N$ then $\operatorname{codim} \alpha \beta_{M}>d$, therefore, $\alpha \beta_{M}=0$. So we can set $\beta=\beta_{N}$.

We fix a homogeneous $\mathbb{Z} / p$-basis $\left\{\alpha_{i}\right\}$ of $R$ and the dual basis $\left\{\alpha_{i}^{\#}\right\}$ with respect to the pairing introduced in Proposition 5.3.

5.4. Corollary. - For $|M| \leq|N|$ we have

$$
\operatorname{deg}\left(e^{M} \alpha_{i} \alpha_{j}^{\#}\right)=\left\{\begin{array}{lc}
1, & M=N \text { and } i=j ; \\
0, & \text { otherwise. }
\end{array}\right.
$$

Proof. - If $M=N$, then it follows from the definition of the dual basis. Assume $|M|<|N|$. If $\operatorname{deg}\left(e^{M} \alpha_{i} \alpha_{j}^{\#}\right) \neq 0$, then $\operatorname{codim}\left(\alpha_{i} \alpha_{j}^{\#}\right)>d$, in contradiction with the fact that $\alpha_{i} \alpha_{j}^{\#} \in R$. Hence, we are reduced to the case $M \neq N$ and $|M|=|N|$. Since $|M|=|N|$, $\operatorname{codim}\left(\alpha_{i} \alpha_{j}^{\#}\right)=d$ and, hence, $R^{+} \alpha_{i} \alpha_{j}^{\#}=0$. On the other hand there exists $i$ such that $m_{i} \geq p^{k_{i}}$ and $e^{p^{k_{i}}} \in \mathrm{Ch}(\overline{\mathfrak{X}}) \cdot R^{+}$. Hence, $e^{M} \alpha_{i} \alpha_{j}^{\#}=0$.

5.5. Definition. - Given two pairs $(L, l)$ and $(M, m)$, where $L, M$ are $r$-tuples and $l, m$ are integers, we say $(L, l) \leq(M, m)$ if either $L \lesseqgtr M$, or in the case $L=M$ we have $l \leq m$. We introduce a filtration on the $\operatorname{ring} \mathrm{Ch}(\overline{\mathfrak{X}})$ as follows:

The $(M, m)$-th term $\mathrm{Ch}(\overline{\mathfrak{X}})_{M, m}$ of the filtration is the $\mathbb{Z} / p$-subspace spanned by the elements $e^{I} \alpha$ with $I \leq M, \alpha \in R$ homogeneous, codim $\alpha \leq m$.

Define the associated graded ring as follows:

$$
A^{*, *}=\bigoplus_{(M, m)} A^{M, m}, \text { where } A^{M, m}=\operatorname{Ch}(\overline{\mathfrak{X}})_{M, m} / \bigcup_{(L, l) \lesseqgtr(M, m)} \operatorname{Ch}(\overline{\mathfrak{X}})_{L, l} .
$$

By Lemma 5.2 if $M \preccurlyeq N$ the graded component $A^{M, m}$ consists of the classes of elements $e^{M} \alpha$ with $\alpha \in R$ and $\operatorname{codim} \alpha=m$. In particular, $\operatorname{rk} A^{M, m}=\operatorname{rk} R^{m}$. Comparing the ranks we see that $A^{M, m}$ is trivial when $M \npreceq N$. 
Consider the subring $\overline{\mathrm{Ch}}(\mathfrak{X})$ of rational cycles with the induced filtration. The associated graded subring will be denoted by $A_{\mathrm{rat}}^{*, *}$. By definition of the $J$-invariant it follows that the elements $e_{i}^{p^{j_{i}}}, i=1, \ldots, r$, belong to $A_{\text {rat }}^{*, *}$.

Similarly, we introduce a filtration on the $\operatorname{ring} \operatorname{Ch}(\overline{\mathfrak{X}} \times \overline{\mathfrak{X}})$ as follows:

The $(M, m)$-th term of the filtration is the $\mathbb{Z} / p$-subspace spanned by the elements $e^{I} \alpha \times$ $e^{L} \beta$ with $I+L \leq M, \alpha, \beta \in R$ homogeneous and $\operatorname{codim} \alpha+\operatorname{codim} \beta \leq m$.

The associated graded ring will be denoted by $B^{*, *}$. By definition $B^{*, *}$ is isomorphic to the tensor product of graded rings $A^{*, *} \otimes_{\mathbb{Z} / p} A^{*, *}$. The graded subring associated to $\overline{\operatorname{Ch}}(\mathfrak{X} \times \mathfrak{X})$ will be denoted by $B_{\text {rat }}^{*, *}$.

5.6. - The key observation is that due to Corollary 5.4 we have

$$
\begin{gathered}
\mathrm{Ch}(\overline{\mathfrak{X}} \times \overline{\mathfrak{X}})_{M, m} \circ \mathrm{Ch}(\overline{\mathfrak{X}} \times \overline{\mathfrak{X}})_{L, l} \subset \mathrm{Ch}(\overline{\mathfrak{X}} \times \overline{\mathfrak{X}})_{M+L-N, m+l-d} \text { and } \\
\left(\mathrm{Ch}(\overline{\mathfrak{X}} \times \overline{\mathfrak{X}})_{M, m}\right)_{\star}\left(\mathrm{Ch}(\overline{\mathfrak{X}})_{L, l}\right) \subset \mathrm{Ch}(\overline{\mathfrak{X}})_{M+L-N, m+l-d}
\end{gathered}
$$

and, therefore, we have a correctly defined composition law

$$
\circ: B^{M, m} \times B^{L, l} \rightarrow B^{M+L-N, m+l-d}
$$

and the realization map (see 1.1)

$$
\star: B^{M, m} \times A^{L, l} \rightarrow A^{M+L-N, m+l-d}
$$

In particular, $B^{N+*, d+*}$ can be viewed as a graded ring with respect to the composition and $(\alpha \circ \beta)_{\star}=\alpha_{\star} \circ \beta_{\star}$. Note also that both operations preserve rationality of cycles.

The proof of the following result is based on the fact that the group $G$ splits over $F(\mathfrak{X})$.

5.7. Lemma. - The classes of the elements $e_{i} \times 1-1 \times e_{i}$ in $B^{*, *}, i=1, \ldots, r$, belong to $B_{\text {rat }}^{*, *}$

Proof. - Fix an $i$. Since $G$ splits over $F(\mathfrak{X}), F(\mathfrak{X})$ is a splitting field of $\mathfrak{X}$ and by Lemma 1.8 there exists a cycle in $\overline{\mathrm{Ch}^{d_{i}}}(\mathfrak{X} \times \mathfrak{X})$ of the form

$$
\xi=e_{i} \times 1+\sum_{s} \mu_{s} \times \nu_{s}+1 \times \mu,
$$

where $\operatorname{codim} \mu_{s}, \operatorname{codim} \nu_{s}<d_{i}$. Then the cycle

$$
\operatorname{pr}_{13}^{*}(\xi)-\operatorname{pr}_{23}^{*}(\xi)=\left(e_{i} \times 1-1 \times e_{i}\right) \times 1+\sum_{s}\left(\mu_{s} \times 1-1 \times \mu_{s}\right) \times \nu_{s}
$$

belongs to $\overline{\mathrm{Ch}}(\mathfrak{X} \times \mathfrak{X} \times \mathfrak{X})$, where $\mathrm{pr}_{i j}$ denotes the projection on the product of the $i$-th and $j$-th factors. Applying Corollary 3.4 to the projection $\operatorname{pr}_{12}: \mathfrak{X} \times \mathfrak{X} \times \mathfrak{X} \rightarrow \mathfrak{X} \times \mathfrak{X}$ we conclude that there exists a (non-canonical) $\mathrm{Ch}(\mathfrak{X} \times \mathfrak{X})$-linear isomorphism $\mathrm{Ch}(\mathfrak{X} \times \mathfrak{X} \times \mathfrak{X}) \simeq$ $\operatorname{Ch}(\mathfrak{X} \times \mathfrak{X}) \otimes \operatorname{Ch}(\overline{\mathfrak{X}})$, where $\operatorname{Ch}(\mathfrak{X} \times \mathfrak{X})$ acts on the left-hand side via $\mathrm{pr}_{12}^{*}$. This gives rise to a $\mathrm{Ch}(\mathfrak{X} \times \mathfrak{X})$-linear retraction $\delta$ to the pull-back map $\operatorname{pr}_{12}^{*}$. Since the construction of the retraction preserves base change, it preserves rationality of cycles. Hence, passing to a splitting field we obtain a rational cycle

$$
\bar{\delta}\left(\operatorname{pr}_{13}^{*}(\xi)-\operatorname{pr}_{23}^{*}(\xi)\right)=e_{i} \times 1-1 \times e_{i}+\sum_{s}\left(\mu_{s} \times 1-1 \times \mu_{s}\right) \bar{\delta}\left(1 \times 1 \times \nu_{s}\right)
$$

whose image in $B_{\text {rat }}^{*, *}$ is $e_{i} \times 1-1 \times e_{i}$.

$4^{\mathrm{e}}$ SÉRIE - TOME $41-2008-\mathrm{N}^{\mathrm{o}} 6$ 
We will write $(e \times 1-1 \times e)^{M}$ for the product $\prod_{i=1}^{r}\left(e_{i} \times 1-1 \times e_{i}\right)^{m_{i}}$ and $\left(\begin{array}{c}M \\ L\end{array}\right)$ for the product of binomial coefficients $\prod_{i=1}^{r}\left(\begin{array}{c}m_{i} \\ l_{i}\end{array}\right)$. We assume that $\left(\begin{array}{c}m_{i} \\ l_{i}\end{array}\right)=0$ if $l_{i}>m_{i}$. In the computations we will extensively use the following two formulae (the first follows directly from Corollary 5.4 and the second one is a well-known binomial identity).

5.8. - Let $\alpha$ be an element of $R^{*}$ and let $\alpha^{\#}$ be its dual with respect to the non-degenerate pairing from 5.3, i.e. $\operatorname{deg}\left(e^{N} \alpha \alpha^{\#}\right)=1$. Then we have

$$
\left((e \times 1-1 \times e)^{M}\left(\alpha^{\#} \times 1\right)\right)_{\star}\left(e^{L} \alpha\right)=\left(\begin{array}{c}
M \\
M+L-N
\end{array}\right)(-1)^{M+L-N} e^{M+L-N} .
$$

Indeed, expanding the brackets in the left-hand side, we obtain

$$
\left(\sum_{I \preccurlyeq M}(-1)^{I}\left(\begin{array}{c}
M \\
I
\end{array}\right) e^{M-I} \alpha^{\#} \times e^{I}\right)_{\star}\left(e^{L} \alpha\right),
$$

and it remains to apply Corollary 5.4.

5.9 (Lucas' Theorem). - The following identity holds

$$
\left(\begin{array}{c}
n \\
m
\end{array}\right) \equiv \prod_{i \geq 0}\left(\begin{array}{c}
n_{i} \\
m_{i}
\end{array}\right) \quad \bmod p
$$

where $m=\sum_{i \geq 0} m_{i} p^{i}$ and $n=\sum_{i \geq 0} n_{i} p^{i}$ are the base $p$ presentations of $m$ and $n$.

Let $J=J_{p}(G)=\left(j_{1}, \ldots, j_{r}\right)$ be the $J$-invariant of $G$ (see Definition 4.6). Set $K=$ $\left(k_{1}, \ldots, k_{r}\right)$.

5.10. Proposition. - Let $\left\{\alpha_{i}\right\}$ be a homogeneous $\mathbb{Z} / p$-basis of $R$. Then the set of elements $\mathcal{B}=\left\{e^{p^{J} L} \alpha_{i} \mid L \preccurlyeq p^{K-J}-1\right\}$ forms a $\mathbb{Z} / p$-basis of $A_{\text {rat }}^{* * *}$.

Proof. - According to Lemma 5.2 the elements from $\mathcal{B}$ are linearly independent. Assume $\mathcal{B}$ does not generate $A_{\text {rat }}^{*, *}$. Choose an element $\omega \in A_{\text {rat }}^{M, m}$ of the smallest index $(M, m)$ which is not in the linear span of $\mathcal{B}$. By definition of $A^{M, m}$ (see Definition 5.5) $\omega$ can be written as $\omega=e^{M} \alpha$, where $M \preccurlyeq N, \alpha \in R^{m}$ and $M$ cannot be presented as $M=p^{J} L^{\prime}$ for an $r$-tuple $L^{\prime}$. The latter means that in the decomposition of $M$ into $p$-primary and $p$-coprimary components $M=p^{S} L$, where $M=\left(m_{1}, \ldots, m_{r}\right), S=\left(s_{1}, \ldots, s_{r}\right), L=\left(l_{1}, \ldots, l_{r}\right)$ and $p \nmid l_{k}$ for $k=1, \ldots, r$, we have $J \npreceq S$. We choose an $i$ such that $s_{i}<j_{i}$. We denote $M_{i}=\left(0, \ldots, 0, m_{i}, 0, \ldots, 0\right)$ and $S_{i}=\left(0, \ldots, 0, s_{i}, 0, \ldots, 0\right)$, where $m_{i}$ and $s_{i}$ stand at the $i$-th place.

Set $T=N-M+M_{i}$. By Lemma 5.7 and 5.8 together with observation 5.6 the element

$$
\left((e \times 1-1 \times e)^{T}\left(\alpha^{\#} \times 1\right)\right)_{\star}\left(e^{M} \alpha\right)=\left(\begin{array}{c}
p^{k_{i}}-1 \\
m_{i}
\end{array}\right)(-1)^{m_{i}} e^{m_{i}}
$$

belongs to $A_{\mathrm{rat}}^{M_{i}, 0}$. By 5.9 we have $p \nmid\left(\begin{array}{c}p^{k_{i}}-1 \\ m_{i}\end{array}\right)$ and, therefore, this element is non-trivial. Moreover, since $s_{i}<j_{i}$, this element is not in the span of $\mathcal{B}$. Since $(M, m)$ was chosen to be the smallest index and $\left(M_{i}, 0\right) \leq(M, m)$ we obtain that $(M, m)=\left(M_{i}, 0\right)$. Repeating the same arguments for $T=N-M_{i}+p^{S_{i}}$ we obtain that $M_{i}=p^{S_{i}}$, i.e. $l_{i}=1$.

Now let $\gamma$ be a representative of $\omega=e_{i}^{p^{s_{i}}}$ in $\overline{\mathrm{Ch}}(\mathfrak{X})$. Then its image $\pi(\gamma)$ in $\mathfrak{C h}(G)$ has the leading term $x_{i}^{p^{s_{i}}}$ with $s_{i}<j_{i}$. This contradicts the definition of the $J$-invariant. 


\subsection{Corollary. - The elements}

$$
\left\{(e \times 1-1 \times e)^{S}\left(e^{p^{J} L} \alpha_{i} \times e^{p^{J}\left(p^{K-J}-1-M\right)} \alpha_{j}^{\#}\right) \mid L, M \preccurlyeq p^{K-J}-1, S \preccurlyeq p^{J}-1\right\}
$$

form $a \mathbb{Z} / p$-basis of $B_{\mathrm{rat}}^{*, *}$. In particular, the ones such that $S=p^{J}-1$ and $L=M$ form a basis of $B_{\mathrm{rat}}^{N, d}$.

Proof. - According to Lemma 5.2 these elements are linearly independent and their number is $p^{|2 K-J|}(\mathrm{rk} R)^{2}$. They are rational by Definition 5.5 and Lemma 5.7. Applying Corollary 3.4 to the projection $\overline{\mathfrak{X}} \times \overline{\mathfrak{X}} \rightarrow \overline{\mathfrak{X}}$ we obtain that

$$
\operatorname{rk} B_{\text {rat }}^{*, *}=\operatorname{rk} \overline{\mathrm{Ch}}(\mathfrak{X} \times \mathfrak{X})=\operatorname{rk} \overline{\mathrm{Ch}}(\mathfrak{X}) \cdot \operatorname{rk} \operatorname{Ch}(\overline{\mathfrak{X}}),
$$

where the latter coincides with rk $A_{\text {rat }}^{*, *} \cdot p^{|K|} \operatorname{rk} R=p^{|2 K-J|}(\mathrm{rk} R)^{2}$ by Lemma 5.2 and Proposition 5.10 .

\subsection{Lemma. - The elements}

$$
\theta_{L, M, i, j}=(e \times 1-1 \times e)^{p^{J}-1}\left(e^{p^{J} L} \alpha_{i} \times e^{p^{J}\left(p^{K-J}-1-M\right)} \alpha_{j}^{\#}\right), L, M \preccurlyeq p^{K-J}-1,
$$

belong to $B_{\text {rat }}^{*, *}$ and satisfy the relations $\theta_{L, M, i, j} \circ \theta_{L^{\prime}, M^{\prime}, i^{\prime}, j^{\prime}}=\delta_{L M^{\prime}} \delta_{i j^{\prime}} \theta_{L^{\prime}, M, i^{\prime}, j}$ and $\sum_{L, i} \theta_{L, L, i, i}=\Delta_{\overline{\mathfrak{X}}}$

Proof. - Expanding the brackets and using the identity $\left(\begin{array}{c}p^{j}-1 \\ i\end{array}\right) \equiv(-1)^{i} \bmod p$, we see that

$$
\theta_{L, M, i, j}=\sum_{I \preccurlyeq p^{J}-1} e^{p^{J} L+I} \alpha_{i} \times e^{N-p^{J} M-I} \alpha_{j}^{\#},
$$

and the composition relation follows from Corollary 5.4. By definition we have

$$
\sum_{L, i} \theta_{L, L, i, i}=\sum_{I \preccurlyeq N, i} e^{I} \alpha_{i} \times e^{N-I} \alpha_{i}^{\#}
$$

By Corollary 5.4 the latter sum acts trivially on all basis elements of $\mathrm{Ch}(\overline{\mathfrak{X}})$ and, hence, coincides with the diagonal.

We are now ready to provide a motivic decomposition of the variety of complete flags.

5.13. TheOREM. - Let $G$ be a semisimple linear algebraic group of inner type over a field $F$ and let $\mathfrak{X}$ be the variety of complete $G$-flags. Let $p$ be a prime. Assume that $J_{p}(G)=\left(j_{1}, \ldots, j_{r}\right)$. Then the motive of $\mathfrak{X}$ is isomorphic to the direct sum

$$
\mathcal{M}(\mathfrak{X} ; \mathbb{Z} / p) \simeq \bigoplus_{i \geq 0} \mathcal{R}_{p}(G)(i)^{\oplus c_{i}}
$$

where the motive $\mathcal{R}_{p}(G)$ is indecomposable, its Poincaré polynomial over a splitting field is given by

$$
P\left(\overline{\mathcal{R}_{p}(G)}, t\right)=\prod_{i=1}^{r} \frac{1-t^{d_{i} p^{j_{i}}}}{1-t^{d_{i}}}
$$

and the integers $c_{i}$ are the coefficients of the quotient

$$
\sum_{i \geq 0} c_{i} t^{i}=P\left(\mathrm{Ch}^{*}(\overline{\mathfrak{X}}), t\right) / P\left(\overline{\mathcal{R}_{p}(G)}, t\right) .
$$

$4{ }^{\mathrm{e}}$ SÉRIE - TOME $41-2008-\mathrm{N}^{\mathrm{o}} 6$ 
Proof. - Consider the projection map

$$
f^{0}: \overline{\mathrm{Ch}}(\mathfrak{X} \times \mathfrak{X})_{N, d} \rightarrow B_{\text {rat }}^{N, d} .
$$

Observe that the kernel of $f^{0}$ is nilpotent. Indeed, any element $\xi$ from ker $f^{0}$ belongs to $\mathrm{Ch}(\overline{\mathfrak{X}} \times \overline{\mathfrak{X}})_{M, m}$ for some $(M, m) \lesseqgtr(N, d)$ which depends on $\xi$. Then by 5.6 its $i$-th composition power $\xi^{\circ i}$ belongs to the graded component $\mathrm{Ch}(\overline{\mathfrak{X}} \times \overline{\mathfrak{X}})_{i M-(i-1) N, i m-(i-1) d}$, and, therefore, becomes trivial for $i$ big enough.

By Lemma 5.12 the elements $\theta_{L, L, i, j}$ form a family of pairwise-orthogonal idempotents whose sum is the identity. Therefore, by Proposition 2.6 there exist pair-wise orthogonal idempotents $\varphi_{L, i}$ in $\overline{\mathrm{Ch}}(\mathfrak{X} \times \mathfrak{X})$ which are mapped to $\theta_{L, L, i, i}$ and whose sum is the identity.

Recall (see 1.1) that given two correspondences $\phi$ and $\psi$ in $\overline{\mathrm{Ch}}(\mathfrak{X} \times \mathfrak{X})$ of degrees $c$ and $c^{\prime}$ respectively its composite $\phi \circ \psi$ has degree $c+c^{\prime}$. Using this fact we conclude that the homogeneous components of $\varphi_{L, i}$ of codimension $\operatorname{dim} \mathfrak{X}$ are pair-wise orthogonal idempotents whose sum is the identity. Hence, we may assume that $\varphi_{L, i}$ belong to $\overline{\mathrm{Ch}^{\operatorname{dim} \mathfrak{X}}}(\mathfrak{X} \times \mathfrak{X})$.

We now show that $\varphi_{L, i}$ are indecomposable. By Corollary 5.11 and Lemma 5.12 the ring $\left(B_{\text {rat }}^{N, d}, \circ\right)$ can be identified with a product of matrix rings over $\mathbb{Z} / p$

$$
B_{\mathrm{rat}}^{N, d} \simeq \prod_{s=0}^{d} \operatorname{End}\left((\mathbb{Z} / p)^{p^{|K-J|} \mathrm{rk} R^{s}}\right) .
$$

By means of this identification $\theta_{L, L, i, i}: e^{p^{J} M} \alpha_{j} \mapsto \delta_{L, M} \delta_{i, j} e^{p^{J} L} \alpha_{i}$ is an idempotent of rank 1 and, therefore, is indecomposable. Since the kernel of $f^{0}$ is nilpotent, the $\varphi_{L, i}$ are indecomposable as well.

Next we show that $\varphi_{L, i}$ is isomorphic to $\varphi_{M, j}$. In the ring $B_{\text {rat }}^{*, *}$ mutually inverse isomorphisms between them are given by $\theta_{L, M, i, j}$ and $\theta_{M, L, j, i}$. Let

$$
f: \overline{\mathrm{Ch}}(\mathfrak{X} \times \mathfrak{X}) \rightarrow B_{\text {rat }}^{*, *}
$$

be the leading term map; it means that for any $\gamma \in \overline{\mathrm{Ch}}(\mathfrak{X} \times \mathfrak{X})$ we find the smallest degree $(I, s)$ such that $\gamma$ belongs to $\overline{\operatorname{Ch}}(\mathfrak{X} \times \mathfrak{X})_{I, s}$ and set $f(\gamma)$ to be the image of $\gamma$ in $B_{\text {rat }}^{I, s}$. Note that $f$ is not a homomorphism but satisfies the condition that $f(\xi) \circ f(\eta)$ equals either $f(\xi \circ \eta)$ or 0 . Choose preimages $\psi_{L, M, i, j}$ and $\psi_{M, L, j, i}$ of $\theta_{L, M, i, j}$ and $\theta_{M, L, j, i}$ by means of $f$. Applying Lemma 2.5 we obtain mutually inverse isomorphisms $\vartheta_{L, M, i, j}$ and $\vartheta_{M, L, j, i}$ between $\varphi_{L, i}$ and $\varphi_{M, j}$. By the definition of $f$ it remains to take their homogeneous components of the appropriate degrees.

Applying now Lemma 2.9 and Corollary 2.10 to the restriction map

$$
\operatorname{res}_{F}: \operatorname{End}(\mathcal{M}(\mathfrak{X} ; \mathbb{Z} / p)) \rightarrow \overline{\operatorname{End}}(\mathcal{M}(\mathfrak{X} ; \mathbb{Z} / p))
$$

and the family of idempotents $\varphi_{L, i}$ we obtain a family of pair-wise orthogonal idempotents $\phi_{L, i} \in \operatorname{End}(\mathcal{M}(\mathfrak{X} ; \mathbb{Z} / p))$ such that

$$
\Delta_{\mathfrak{X}}=\sum_{L, i} \phi_{L, i} .
$$

Since $\operatorname{res}_{F_{s} / F}$ lifts isomorphisms, for the respective motives we have

$$
\left(\mathfrak{X}, \phi_{L, i}\right) \simeq\left(\mathfrak{X}, \phi_{0,0}\right)\left(|L|+\operatorname{codim} \alpha_{i}\right)
$$


for all $L$ and $i$ (see 2.2). The twists $|L|+\operatorname{codim} \alpha_{i}$ can be easily recovered from the explicit formula for $\theta_{L, L, i, i}$ (see Lemma 5.12). Denoting $\mathcal{R}_{p}(G)=\left(\mathfrak{X}, \phi_{0,0}\right)$ we obtain the desired motivic decomposition.

Finally, consider the motive $\mathcal{R}_{p}(G)$ over a splitting field. The idempotent $\theta_{0,0,0,0}$ splits into the sum of pair-wise orthogonal (non-rational) idempotents $e^{I} \times e^{N-I} 1^{\#}, I \preccurlyeq p^{J}-1$. The motive corresponding to each summand is isomorphic to $(\mathbb{Z} / p)(|I|)$. Therefore, we obtain the decomposition into Tate motives

$$
\overline{\mathcal{R}_{p}(G)} \simeq \bigoplus_{I \preccurlyeq p^{J}-1}(\mathbb{Z} / p)(|I|),
$$

which gives formula (3) for the Poincaré polynomial.

As a direct consequence of the proof we obtain

5.14. Corollary. - Any direct summand of $\mathcal{M}(\mathfrak{X} ; \mathbb{Z} / p)$ is isomorphic to a direct sum of twisted copies of $\mathcal{R}_{p}(G)$.

Proof. - Indeed, in the ring $B_{\mathrm{rat}}^{N, d}$ any idempotent is isomorphic to a sum of idempotents $\theta_{L, L, i, i}$, and the map $f^{0}$ lifts isomorphisms.

5.15. Remark. - Corollary 5.14 can be viewed as a particular case of the Krull-Schmidt Theorem proven by V. Chernousov and A. Merkurjev (see [8, Corollary 9.7]).

5.16. Definition. - Let $G$ be a linear algebraic group over a field $F$ and let $X$ be a projective homogeneous $G$-variety. We say $X$ is generically split if the group $G$ splits over the generic point of $X$.

The main result of the present paper is the following

5.17. THEOREM. - Let $G$ be a semisimple linear algebraic group of inner type over a field $F$ and let $p$ be a prime integer. Let $X$ be a generically split projective homogeneous $G$-variety. Then the motive of $X$ with $\mathbb{Z} / p$-coefficients is isomorphic to the direct sum

$$
\mathcal{M}(X ; \mathbb{Z} / p) \simeq \bigoplus_{i \geq 0} \mathcal{R}_{p}(G)(i)^{\oplus a_{i}}
$$

where $\mathcal{R}_{p}(G)$ is an indecomposable motive; Poincaré polynomial $P\left(\overline{\mathcal{R}_{p}(G)}, t\right)$ is given by (3) and, hence, only depends on the $J$-invariant of $G$; the $a_{i}$ 's are the coefficients of the quotient polynomial

$$
\sum_{i \geq 0} a_{i} t^{i}=P\left(\mathrm{CH}^{*}(\bar{X}), t\right) / P\left(\overline{\mathcal{R}_{p}(G)}, t\right)
$$

Proof. - Let $\mathfrak{X}$ be the variety of complete $G$-flags. According to Theorem 3.7 the motive of $Y=\mathfrak{X}$ is isomorphic to a direct sum of twisted copies of the motive of $X$. To finish the proof we apply Theorem 5.13 and Corollary 5.14.

We now provide several properties of $\mathcal{R}_{p}(G)$ which will be extensively used in the applications.

5.18. Proposition. - Let $G, G^{\prime}$ be two semisimple algebraic groups of inner type over $F$ and let $\mathfrak{X}, \mathfrak{X}^{\prime}$ be the corresponding varieties of complete flags.

$4^{\mathrm{e}}$ SÉRIE - TOME $41-2008-\mathrm{N}^{\mathrm{o}} 6$ 
(i) (base change) For any field extension $E / F$ we have

$$
\mathcal{R}_{p}(G)_{E} \simeq \bigoplus_{i \geq 0} \mathcal{R}_{p}\left(G_{E}\right)(i)^{\oplus a_{i}}
$$

where $\sum a_{i} t^{i}=P\left(\overline{\mathcal{R}_{p}(G)}, t\right) / P\left(\overline{\mathcal{R}_{p}\left(G_{E}\right)}, t\right)$.

(ii) (transfer argument) If $E / F$ is a field extension of degree coprime to $p$ then $J_{p}\left(G_{E}\right)=$ $J_{p}(G)$ and $\mathcal{R}_{p}\left(G_{E}\right)=\mathcal{R}_{p}(G)_{E}$. Moreover, if $\mathcal{R}_{p}\left(G_{E}\right) \simeq \mathcal{R}_{p}\left(G_{E}^{\prime}\right)$ then $\mathcal{R}_{p}(G) \simeq$ $\mathcal{R}_{p}\left(G^{\prime}\right)$.

(iii) (comparison lemma) If $G$ splits over $F\left(\mathfrak{X}^{\prime}\right)$ and $G^{\prime}$ splits over $F(\mathfrak{X})$ then $\mathcal{R}_{p}(G) \simeq$ $\mathcal{R}_{p}\left(G^{\prime}\right)$

Proof. - The first claim follows from Theorem 5.13 and Corollary 5.14. To prove the second claim note that $E$ is rank preserving with respect to $\mathfrak{X}$ and $\mathfrak{X} \times \mathfrak{X}$ by Lemma 1.12. Now $J_{p}\left(G_{E}\right)=J_{p}(G)$ by Lemma 1.10 , and hence $\mathcal{R}_{p}\left(G_{E}\right)=\mathcal{R}_{p}(G)_{E}$ by the first claim. The remaining part of the claim follows from Corollary 2.11 applied to the variety $\mathfrak{X} \amalg \mathfrak{X}^{\prime}$.

We now prove the last claim. The variety $\mathfrak{X} \times \mathfrak{X}^{\prime}$ is the variety of complete $G \times G^{\prime}$-flags. By Corollary 3.4 applied to the projections $\mathfrak{X} \times \mathfrak{X}^{\prime} \rightarrow \mathfrak{X}$ and $\mathfrak{X} \times \mathfrak{X}^{\prime} \rightarrow \mathfrak{X}^{\prime}$ we can express $\mathcal{M}\left(\mathfrak{X} \times \mathfrak{X}^{\prime} ; \mathbb{Z} / p\right)$ in terms of $\mathcal{M}(\mathfrak{X} ; \mathbb{Z} / p)$ and $\mathcal{M}\left(\mathfrak{X}^{\prime} ; \mathbb{Z} / p\right)$. The latter motives can be expressed in terms of $\mathcal{R}_{p}(G)$ and $\mathcal{R}_{p}\left(G^{\prime}\right)$. The claim now follows from the Krull-Schmidt theorem (see Corollary 5.14).

5.19. Corollary. - We have $\mathcal{R}_{p}(G) \simeq \mathcal{R}_{p}\left(G_{a n}\right)$, where $G_{a n}$ is the semisimple anisotropic kernel of $G$.

Finally, we provide conditions which allow one to lift a motivic decomposition of a generically split homogeneous variety with $\mathbb{Z} / m$-coefficients to a decomposition with $\mathbb{Z}$-coefficients.

5.20. Definition. - Let $m$ be a positive integer. We say a polynomial $g(t)$ is $m$-positive, if $g \neq 0, P\left(\overline{\mathcal{R}_{p}(G)}, t\right) \mid g(t)$ and the quotient polynomial $g(t) / P\left(\overline{\mathcal{R}_{p}(G)}, t\right)$ has non-negative coefficients for all primes $p$ dividing $m$.

5.21. Proposition. - Let $G$ be a semisimple linear algebraic group of inner type over a field $F$ and let $X$ be a generically split projective homogeneous $G$-variety. Assume that $X$ splits by a field extension of degree $m$. Let $f(t)$ be an m-positive polynomial dividing $P(\mathcal{M}(\bar{X}), t)$ which cannot be presented as a sum of two m-positive polynomials. Then the motive of $X$ with integer coefficients splits as a direct sum

$$
\mathcal{M}(X ; \mathbb{Z}) \simeq \bigoplus_{i} \mathcal{R}_{i}\left(c_{i}\right), \quad c_{i} \in \mathbb{Z}
$$

where $\mathcal{R}_{i}$ are indecomposable and $P\left(\overline{\mathcal{R}}_{i}, t\right)=f(t)$ for all $i$. Moreover, if $m=2,3,4$ or 6 , then all motives $\mathcal{R}_{i}$ are isomorphic up to twists.

Proof. - First, we apply Corollary 2.7 to obtain a decomposition with $\mathbb{Z} / m$-coefficients. By Lemma 1.12 our field extension is rank preserving so we can apply Theorem 2.16 to lift the decomposition to the category of motives with $\mathbb{Z}$-coefficients. 


\section{Applications of the $J_{p}$ and of the motive $\mathcal{R}_{p}$}

Let $G$ be a semisimple group of inner type over $F$ and let $\mathfrak{X}$ be the variety of complete $G$-flags.

First, we obtain the following expression for the canonical p-dimension of $\mathfrak{X}$ (see [13, §90]).

6.1. Proposition. - In the notation of Theorem 5.13 we have

$$
\operatorname{cd}_{p}(\mathfrak{X})=\sum_{i=1}^{r} d_{i}\left(p^{j_{i}}-1\right) .
$$

Proof. - Follows from Proposition 5.10 and [22, Theorem 5.8].

Let $X$ be a smooth projective variety which has a splitting field.

6.2. Lemma. - For any $\phi, \psi \in \mathrm{CH}^{*}(\bar{X} \times \bar{X})$ one has

$$
\operatorname{deg}\left(\left(\operatorname{pr}_{2}\right)_{*}\left(\phi \cdot \psi^{t}\right)\right)=\operatorname{tr}\left((\phi \circ \psi)_{\star}\right) .
$$

Proof. - Choose a homogeneous basis $\left\{e_{i}\right\}$ of $\mathrm{CH}^{*}(\bar{X})$. Let $\left\{e_{i}^{\vee}\right\}$ be its Poincaré dual. Since both sides of the relation under proof are bilinear, it suffices to check the assertion for $\phi=e_{i} \times e_{j}^{\vee}$ and $\psi=e_{k} \times e_{l}^{\vee}$. In this case both sides of the relation are equal to $\delta_{i l} \delta_{j k}$.

We denote the greatest common divisor of degrees of all zero cycles on $X$ by $d(X)$ and its $p$-primary component by $d_{p}(X)$.

6.3. Corollary. - Let $m$ be an integer. For any $\phi \in \overline{\mathrm{CH}}(X \times X ; \mathbb{Z} / m)$ we have

$$
\operatorname{gcd}(d(X), m) \mid \operatorname{tr}\left(\phi_{\star}\right) .
$$

Proof. - Set $\psi=\Delta_{\bar{X}}$ and apply Lemma 6.2.

6.4. Corollary. - Assume that $\mathcal{M}(X ; \mathbb{Z} / p)$ has a direct summand $M$. Then

- $d_{p}(X) \mid P(\bar{M}, 1)$;

- if $d_{p}(X)=P(\bar{M}, 1)$ and the kernel of the restriction $\operatorname{End}(\mathcal{M}(X)) \rightarrow \operatorname{End}(\mathcal{M}(\bar{X}))$ consists of nilpotents, then $M$ is indecomposable.

Proof. - Set $q=d_{p}(X)$ for brevity. Let $M=(X, \phi)$. By Corollary 2.7 there exists an idempotent $\varphi \in \operatorname{End}(\mathcal{M}(X) ; \mathbb{Z} / q)$ such that $\varphi \bmod p=\phi$. Then $\operatorname{res}(\varphi) \in$ $\operatorname{End}(\mathcal{M}(\bar{X}) ; \mathbb{Z} / q)$ is a rational idempotent. Since every projective module over $\mathbb{Z} / q$ is free, we have

$$
\operatorname{tr}\left(\operatorname{res}(\varphi)_{\star}\right)=\operatorname{rk}_{\mathbb{Z} / q}\left(\operatorname{res}(\varphi)_{\star}\right)=\operatorname{rk}_{\mathbb{Z} / p}\left(\operatorname{res}(\phi)_{\star}\right)=P(\bar{M}, 1) \quad \bmod q
$$

and the first claim follows from Corollary 6.3. The second claim follows from the first one, since the second assumption implies that for any non-trivial direct summand $M^{\prime}$ of $M$ we have $P\left(\overline{M^{\prime}}, 1\right)<P(\bar{M}, 1)$.

6.5. - Let $n(G)$ denote the greatest common divisor of degrees of all finite splitting fields of $G$ and let $n_{p}(G)$ be its $p$-primary component. Note that $n(G)=d(\mathfrak{X})$ and $n_{p}(G)=d_{p}(\mathfrak{X})$.

We obtain the following estimate on $n_{p}(G)$ in terms of the $J$-invariant (cf. [13, Prop. 88.11] in the case of quadrics).

$4^{\text {e }}$ SÉRIE - TOME $41-2008-\mathrm{N}^{\mathrm{o}} 6$ 
6.6. Proposition. - Let $G$ be a semisimple linear algebraic group of inner type with $J_{p}(G)=\left(j_{1}, \ldots, j_{r}\right)$. Then

$$
n_{p}(G) \leq p \sum_{i} j_{i}
$$

Proof. - Follows from Theorem 5.13 and Corollary 6.4.

6.7. COROLLARY. - The following statements are equivalent:

- $J_{p}(G)=(0, \ldots, 0)$;

- $n_{p}(G)=1$;

- $\mathcal{R}_{p}(G)=\mathbb{Z} / p$.

Proof. - If $J_{p}(G)=(0, \ldots, 0)$ then $n_{p}(G)=1$ by Proposition 6.6. If $n_{p}(G)=1$ then there exists a splitting field $L$ of degree $m$ prime to $p$ and, therefore, $\mathcal{R}_{p}(G)=\mathbb{Z} / p$ by transfer argument 5.18(ii). The remaining implication is obvious.

\section{Examples}

In the present section we provide examples of motivic decompositions of projective homogeneous $G$-varieties obtained using Theorem 5.17, where $G$ is a simple group of inner type over a field $F$. We identify certain groups of small ranks via exceptional isomorphisms, e.g. $\mathrm{B}_{2}=\mathrm{C}_{2}$ (see $\left.[24, \S 15]\right)$.

7.1. The case $r=d_{1}=1$.

According to Table $4.13 J_{p}(G)=\left(j_{1}\right)$ for some $j_{1} \geq 0$ if and only if $G_{0}$ is a split group of type $\mathrm{A}_{n}$ or $\mathrm{C}_{n}$. In this case $p \mid(n+1)$ or $p=2$ respectively.

Let $G={ }_{\xi} G_{0}$ by means of $\xi \in Z^{1}\left(F, G_{0}\right)$. Let $D$ be a central division algebra of degree $d$ corresponding to the class of the image of $\xi$ in $H^{1}\left(F\right.$, Aut $\left.G_{0}\right)$ (see [24, p. 396 and p. 404]). Observe that $p \mid d$.

Let $X_{\Theta}$ be a projective homogeneous $G$-variety given by a subset $\Theta$ of vertices of the respective Dynkin diagram $\mathcal{D}$. By [34, p.55-56] $X_{\Theta}$ is generically split if $\mathcal{D} \backslash \Theta$ contains at least one $r$-th vertex with $p \nmid r$ (cf. Example 3.6). Observe that the converse is also true by the Index Reduction Formula (see [28, Appendix I, III]).

Then by Theorem 5.17 we obtain that for a generically split $X_{\Theta}$

$$
\mathcal{M}\left(X_{\Theta} ; \mathbb{Z} / p\right) \simeq \bigoplus_{i \geq 0} \mathcal{R}_{p}(G)(i)^{\oplus a_{i}}
$$

where $\mathcal{R}_{p}(G)$ is indecomposable and

$$
\overline{\mathcal{R}_{p}(G)} \simeq \bigoplus_{i=0}^{p^{j_{1}}-1}(\mathbb{Z} / p)(i) .
$$

We now identify $\mathcal{R}_{p}(G)$. Using the comparison lemma (see Proposition 5.18) we conclude that $\mathcal{R}_{p}(G)$ only depends on $D$, so $p^{j_{1}} \mid d$. On the other hand by Proposition 6.6 we have $n_{p}(G) \leq p^{j_{1}}$. Since $n_{p}(G)$ is the $p$-primary part of $d$, it coincides with $p^{j_{1}}$ (in the $\mathrm{C}_{n}$-case it coincides with $d$ ).

We have $D \simeq D_{p} \otimes_{F} D^{\prime}$, where $p^{j_{1}}=\operatorname{ind}\left(D_{p}\right)$ and $p \nmid \operatorname{ind}\left(D^{\prime}\right)$. Passing to a splitting field of $D^{\prime}$ of degree prime to $p$ and using Proposition 5.18 we conclude that the motives of 
$X_{\Theta}$ and $\operatorname{SB}\left(D_{p}\right)$ are direct sums of twisted $\mathcal{R}_{p}(G)$. Comparing the Poincaré polynomials we conclude that

7.1. LemmA. - $\mathcal{M}\left(\mathrm{SB}\left(D_{p}\right) ; \mathbb{Z} / p\right) \simeq \mathcal{R}_{p}(G)$.

7.2. Corollary. - The motive of $\mathrm{SB}(D)$ with integer coefficients is indecomposable.

Proof. - We apply Proposition 5.21 to $X=\mathrm{SB}(D)$ and compare the Poincaré polynomials of $\mathcal{M}(X)$ and $\mathcal{R}_{i}$.

7.3. REMARK. - Indeed, we provided a uniform proof of the results of paper [21]. Namely, the decomposition of $\mathcal{M}\left(\mathrm{SB}\left(M_{m}(D)\right) ; \mathbb{Z} / p\right)$ (see [21, Cor. 1.3.2]) and indecomposability of $\mathcal{M}(\mathrm{SB}(D) ; \mathbb{Z})$ (see [21, Thm. 2.2.1]).

7.2. The case $r=1$ and $d_{1}>1$.

According to Table 4.13 this holds if and only if $G_{0}$ is a split group of type (here ${ }^{\text {sc }}$ denotes a simply-connected group)

- $p=2: \mathrm{G}_{2}, \mathrm{~F}_{4}, \mathrm{E}_{6}, \mathrm{~B}_{3}^{\mathrm{sc}}, \mathrm{B}_{4}^{\mathrm{sc}}, \mathrm{D}_{4}^{\mathrm{sc}}, \mathrm{D}_{5}^{\mathrm{sc}}$;

- $p=3: \mathrm{F}_{4}, \mathrm{E}_{7}, \mathrm{E}_{6}^{\mathrm{sc}}$;

- $p=5: \mathrm{E}_{8}$.

Moreover, it follows from Table 4.13 that in all these cases $k_{1}=1$.

Let $G={ }_{\xi} G_{0}$, where $\xi \in Z^{1}\left(F, G_{0}\right)$. Let $X$ be a generically split projective homogeneous $G$-variety (cf. Example 3.6). By Theorem 5.17 we obtain the decomposition

$$
\mathcal{M}(X ; \mathbb{Z} / p) \simeq \bigoplus_{i \geq 0} \mathcal{R}_{p}(G)(i)^{\oplus a_{i}}
$$

where the motive $\mathcal{R}_{p}(G)$ is indecomposable and (cf. [37, (5.4-5.5)])

$$
\overline{\mathcal{R}_{p}(G)} \simeq \bigoplus_{i=0}^{p-1}(\mathbb{Z} / p)(i \cdot(p+1)) .
$$

We now identify $\mathcal{R}_{p}(G)$.

Observe that for any such group $G$ there exists a finite field extension $E / F$ of degree coprime to $p$ such that $G_{E}={ }_{\xi} G_{0, E}$, where $\xi \in Z^{1}\left(E, G_{0}^{\mathrm{sc}}\right)$. Indeed, for $\mathrm{E}_{6}(p=2)$ and $\mathrm{E}_{7}$ $(p=3)$ it follows from the fact that the center of $\mathrm{E}_{6}^{\mathrm{sc}}$ is $\mu_{3}$ and the center of $\mathrm{E}_{7}^{\mathrm{sc}}$ is $\mu_{2}$. For all other groups it is obvious.

Let $\mathfrak{r}$ be the Rost invariant as defined in [27]. According to [15, Lemma 2.1] if a group is given by a cocycle with values in a simply-connected group, the invariant $\mathfrak{r}$ depends only on the isomorphism class of the group but not on the particular choice of the cocycle. Therefore, the invariant $\mathfrak{r}(G)=\mathfrak{r}(\xi)$ is well-defined over $E$.

Let $\mathfrak{r}_{p}$ be the $p$-component of $\mathfrak{r}$. Observe that for the group $G$ over $E$ the invariant $\mathfrak{r}_{p}$ takes values in $H^{3}\left(E, \mu_{p}^{\otimes 2}\right)$. Define $\mathfrak{r}_{p}(G)=\frac{1}{[E: F]} \operatorname{cores}_{E / F} \mathfrak{r}_{p}\left(G_{E}\right)$. It is easy to see that $\mathfrak{r}_{p}(G)$ does not depend on the choice of $E$. Indeed, if $E$ and $E^{\prime}$ are two such field extensions, we can pass to the composite field $E \cdot E^{\prime}$ and use the functoriality of $\mathfrak{r}_{p}$.

7.4. Lemma. - Let $G$ be a simple linear algebraic group of inner type over $F$ satisfying $r=1$ and $d_{1}>1$ and let $p$ be a prime. Then $\mathfrak{r}_{p}(G)$ is trivial iff $\mathcal{R}_{p}(G) \simeq \mathbb{Z} / p$. 
Proof. - According to [14, Theorem 0.5], [6] and [16, Théorème 10] the invariant $\mathfrak{r}_{p}(G)$ is trivial iff the group $G$ splits over a $p$-primary closure of $F$. By Corollary 6.7 the latter is equivalent to the fact that $\mathcal{R}_{p}(G) \simeq \mathbb{Z} / p$.

7.5. Lemma. - Let $G$ and $G^{\prime}$ be simple linear algebraic groups of inner type over $F$ satisfying $r=1$ and $d_{1}>1$ (observe that in this case $k=1$ ).

If $\mathfrak{r}_{p}(G)=c \cdot \mathfrak{r}_{p}\left(G^{\prime}\right)$ for some $c \in(\mathbb{Z} / p)^{\times}$, then $\mathcal{R}_{p}(G) \simeq \mathcal{R}_{p}\left(G^{\prime}\right)$.

Proof. - By transfer arguments (see Proposition 5.18) it is enough to prove this over a $p$-primary closure of $F$. Let $\mathfrak{X}$ and $\mathfrak{X}^{\prime}$ be the respective varieties of complete flags. Observe that the invariant $\mathfrak{r}_{p}(G)$ becomes trivial over the function field $F(\mathfrak{X})$. Since $\mathfrak{r}_{p}(G)=c \cdot \mathfrak{r}_{p}\left(G^{\prime}\right)$, the invariant becomes trivial over $F\left(\mathfrak{X}^{\prime}\right)$ as well. By Lemma 7.4 $\mathfrak{X}$ splits over $F\left(\mathfrak{X}^{\prime}\right)$. Similarly, $\mathfrak{X}^{\prime}$ splits over $F(\mathfrak{X})$.

Therefore, by Lemma 1.8 there exists a rational cycle $\phi$ in $\mathrm{Ch}_{\operatorname{dim} \mathfrak{X}}\left(\overline{\mathfrak{X}} \times \overline{\mathfrak{X}}^{\prime}\right)$ of the form $\phi=1 \times p t+\sum_{\operatorname{codim} \alpha_{i}>0} \alpha_{i} \times \beta_{i}$. Observe that by definition $\phi_{\star}: p t_{\mathfrak{X}} \mapsto p t_{\mathfrak{X}^{\prime}}$. Similarly, interchanging $\mathfrak{X}$ and $\mathfrak{X}^{\prime}$ we obtain a rational cycle $\phi^{\prime} \in \mathrm{Ch}_{\operatorname{dim} \mathfrak{X}^{\prime}}\left(\mathfrak{X}^{\prime} \times \mathfrak{X}\right)$ such that $\phi_{\star}^{\prime}: p t_{\mathfrak{X}^{\prime}} \mapsto$ $p t_{\mathfrak{X}}$. Restricting $\phi$ and $\phi^{\prime}$ to the direct summands $\overline{\mathcal{R}_{p}(G)}$ and $\overline{\mathcal{R}_{p}\left(G^{\prime}\right)}$ of $\mathcal{M}(\overline{\mathfrak{X}})$ and $\mathcal{M}\left(\overline{\mathfrak{X}}^{\prime}\right)$ respectively we obtain the rational maps $\phi_{R}: \overline{\mathcal{R}_{p}(G)} \rightarrow \overline{\mathcal{R}_{p}\left(G^{\prime}\right)}$ and $\phi_{R}^{\prime}: \overline{\mathcal{R}_{p}\left(G^{\prime}\right)} \rightarrow \overline{\mathcal{R}_{p}(G)}$.

Since the motive $\mathcal{R}_{p}(G)$ is indecomposable and $\operatorname{rk~Ch}^{i}\left(\overline{\mathcal{R}_{p}(G)}\right) \leq 1$ for all $i$, the ring of rational endomorphisms of $\overline{\mathcal{R}_{p}(G)}$ is generated by the identity endomorphism $\Delta$. The same holds for the ring of rational endomorphisms of $\overline{\mathcal{R}_{p}\left(G^{\prime}\right)}$. Since $\left(\phi_{R}^{\prime}\right)_{\star} \circ\left(\phi_{R}\right)_{\star}: p t_{\mathfrak{X}} \mapsto p t_{\mathfrak{X}}$, the composition $\phi_{R}^{\prime} \circ \phi_{R}=\Delta$. Similarly we obtain $\phi_{R} \circ \phi_{R}^{\prime}=\Delta^{\prime}$. By the Rost nilpotence since $\phi_{R}$ and $\phi_{R}^{\prime}$ are rational, the motives $\mathcal{R}_{p}(G)$ and $\mathcal{R}_{p}\left(G^{\prime}\right)$ are isomorphic.

\section{3. $\mathbb{Z}$-coefficients.}

Let $G={ }_{\xi} G_{0}$ be a twisted form by means of a cocycle $\xi \in Z^{1}\left(F, G_{0}\right)$, where $G_{0}$ is a group of type $\mathrm{F}_{4}$ or $\mathrm{E}_{6}^{\mathrm{sc}}$. Assume that $G$ is not split by a field extension of degree less than 4 . Observe that such a group always splits by an extension of degree 6 .

Let $X$ be a generically split projective homogeneous $G$-variety. Then according to Proposition 5.21 the Chow motive of $X$ with integer coefficients splits as a direct sum of twisted copies of an indecomposable motive $\mathcal{R}(G)$ such that

$$
\begin{array}{rlrl}
\mathcal{R}(G) \otimes \mathbb{Z} / 2= & \bigoplus_{i=0,1,2,6,7,8} \mathcal{R}_{2}(G)(i), & P\left(\overline{\mathcal{R}_{2}(G)}, t\right)=1+t^{3}, \\
\mathcal{R}(G) \otimes \mathbb{Z} / 3=\bigoplus_{i=0,1,2,3} \mathcal{R}_{3}(G)(i), & P\left(\overline{\mathcal{R}_{3}(G)}, t\right)=1+t^{4}+t^{8}, \\
P(\overline{\mathcal{R}(G)}, t)=1+t+t^{2}+\cdots+t^{11} . &
\end{array}
$$

7.6. REMARK. - In particular, we provided a uniform proof of the main results of papers [2] and [30], where the cases of $\mathrm{G}_{2}$ - and $\mathrm{F}_{4}$-varieties were considered.

\subsection{The case $r>1$.}

According to Table 4.13 this holds for split groups $G_{0}$ of remaining classical types $\mathrm{B}_{n}, \mathrm{D}_{n}$ for $p=2$; exceptional types $\mathrm{E}_{7}, \mathrm{E}_{8}$ for $p=2$ and $\mathrm{E}_{6}^{\text {ad }}, \mathrm{E}_{8}$ for $p=3$. 


\subsection{Projective Quadric.}

Consider a generically split projective quadric $X$. By $[13,25.6,28.2]$ a quadric $X$ is generically split if and only if it is a Pfister quadric or its codimension one neighbor. Let $\phi$ be the $k$-fold Pfister form (or its codimension one neighbor) defining $X$. Then $X=X_{\Theta}$ is a projective homogeneous $G$-variety, where $G=\mathrm{O}^{+}(\phi)$ is the orthogonal group of $\phi$, and $\Theta$ is the subset of the respective Dynkin diagram obtained by removing the first vertex.

Assume $J_{2}(G) \neq(0, \ldots, 0)$. In view of Corollary 6.7 this holds if and only if $n_{2}(G) \neq 1$. By Springer's Theorem the latter holds if and only if $\phi$ is not split. By Theorem 5.17 we obtain the decomposition

$$
\mathcal{M}(X ; \mathbb{Z} / 2) \simeq \bigoplus_{i \geq 0} \mathcal{R}_{2}(G)(i)^{\oplus a_{i}}
$$

where the motive $\mathcal{R}_{2}(G)$ is indecomposable. Moreover, by Theorem 2.16 the same decomposition holds with $\mathbb{Z}$-coefficients.

We now compute $J_{2}(G)$. Observe that the group $G$ splits over the function field $F(X)$ and $X$ splits over $F(x)$ for any $x \in X$. It is known (see [13, §72]) that the Chow groups $\operatorname{Ch}^{l}(\bar{X})$ for $l<2^{k-1}-1$ consist of rational cycles, i.e. the restriction maps $\mathrm{Ch}^{l}(X) \rightarrow \mathrm{Ch}^{l}\left(X_{F(x)}\right)$ are surjective for $l<2^{k-1}-1$. Then by Proposition 4.9 and Table 4.13 we obtain that $j_{i}=0$ for $1 \leq i<2^{k-2}$. Therefore,

$$
J_{2}(G)=(0, \ldots, 0,1) \text { and } P\left(\overline{\mathcal{R}_{2}(G)}, t\right)=1+t^{2^{k-1}-1} .
$$

Finally, by Corollary 5.14 the motive $\mathcal{R}_{2}(G)$ coincides with the motive introduced in [32], called the Rost motive.

In this way we obtain the Rost decomposition of the motive of a Pfister quadric and its codimension one neighbor.

\subsection{Maximal orthogonal Grassmannian.}

Let $X$ be a connected component of a maximal orthogonal Grassmannian. Then $X=$ $X_{\Theta}$ is a projective homogeneous $G$-variety, where $G=\mathrm{O}^{+}(q)$ is the orthogonal group of $q$, and $\Theta$ is the subset of the respective Dynkin diagram obtained by removing the last vertex. According to [34, p. 55-56] $X$ is generically split.

By Theorem 5.17 we obtain the decomposition

$$
\mathcal{M}(X ; \mathbb{Z} / 2) \simeq \bigoplus_{i \geq 0} \mathcal{R}_{2}(G)(i)^{\oplus a_{i}}
$$

where the motive $\mathcal{R}_{2}(G)$ is indecomposable. Comparing the Poincare polynomials of $\mathcal{M}(X ; \mathbb{Z} / 2)$ and $\mathcal{R}_{2}(G)$ we obtain the following two extreme cases:

- If the group $G$ is given by a generic cocycle, i.e. $q$ is generic, the motive $\mathcal{M}(X ; \mathbb{Z} / 2)$ coincides with $\mathcal{R}_{2}(G)$ and, therefore, is indecomposable. This corresponds to the maximal value of the $J$-invariant.

- If $q=\phi$ is a Pfister form or its codimension one neighbor, then by the previous example $\mathcal{R}_{2}(G)$ coincides with the Rost motive. This corresponds to the minimal non-trivial value of the $J$-invariant (6). 


\section{Acknowledgements}

We are very grateful to Anthony Bak, Fabien Morel and Ivan Panin for an encouraging attention to our work. We would like to thank Mathieu Florence, Skip Garibaldi, Stefan Gille and Nikita Karpenko for many useful discussions on motives, torsors and algebraic groups. We are also grateful to the anonymous referee for numerous comments which helped very much to improve the presentation of the paper.

The essential part of this work formed the PhD Thesis of Victor Petrov (Bielefeld, 2007). We are very grateful to CNRS, DAAD A/04/00348, DFG GI706/1-1, INTAS 05-10000088118 and SFB 701 for financial support.

\section{REFERENCES}

[1] F. W. Anderson, K. R. Fuller, Rings and categories of modules, second ed., Graduate Texts in Math. 13, Springer, 1992.

[2] J.-P. Bonnet, Un isomorphisme motivique entre deux variétés homogènes projectives sous l'action d'un groupe de type $G_{2}$, Doc. Math. 8 (2003), 247-277.

[3] P. Brosnan, Steenrod operations in Chow theory, Trans. Amer. Math. Soc. 355 (2003), 1869-1903.

[4] P. Brosnan, On motivic decompositions arising from the method of Białynicki-Birula, Invent. Math. 161 (2005), 91-111.

[5] B. Calmès, V. Petrov, N. Semenov, K. Zainoulline, Chow motives of twisted flag varieties, Compos. Math. 142 (2006), 1063-1080.

[6] V. Chernousov, A remark on the $(\bmod 5)$-invariant of Serre for groups of type $E_{8}$, Mat. Zametki 56 (1994), 116-121, 157.

[7] V. Chernousov, S. Gille, A. Merkurjev, Motivic decomposition of isotropic projective homogeneous varieties, Duke Math. J. 126 (2005), 137-159.

[8] V. Chernousov, A. Merkurjev, Motivic decomposition of projective homogeneous varieties and the Krull-Schmidt theorem, Transform. Groups 11 (2006), 371-386.

[9] M. Demazure, Invariants symétriques entiers des groupes de Weyl et torsion, Invent. Math. 21 (1973), 287-301.

[10] M. Demazure, Désingularisation des variétés de Schubert généralisées, Ann. Sci. École Norm. Sup. 7 (1974), 53-88.

[11] H. Duan, X. Zhao, A unified formula for Steenrod operations in flag manifolds, Compos. Math. 143 (2007), 257-270.

[12] D. Edidin, W. Graham, Characteristic classes in the Chow ring, J. Algebraic Geom. 6 (1997), 431-443.

[13] R. Elman, N. Karpenko, A. Merkurjev, The algebraic and geometric theory of quadratic forms, to appear in AMS Colloquium Publications.

[14] R. S. Garibaldi, The Rost invariant has trivial kernel for quasi-split groups of low rank, Comment. Math. Helv. 76 (2001), 684-711.

[15] R. S. Garibaldi, H. P. Petersson, Groups of outer type $E_{6}$ with trivial Tits algebras, Transform. Groups 12 (2007), 443-474. 
[16] P. Gille, Invariants cohomologiques de Rost en caractéristique positive, $K$-Theory 21 (2000), 57-100.

[17] A. Grothendieck, La torsion homologique et les sections rationnelles, in Anneaux de Chow et applications, Séminaire C. Chevalley, $2^{\mathrm{e}}$ année, 1958.

[18] A. J. Hahn, O. T. O’Meara, The classical groups and K-theory, Grund. Math. Wiss. 291, Springer, 1989.

[19] H. Hiller, Geometry of Coxeter groups, Research Notes in Math. 54, Pitman (Advanced Publishing Program), 1982.

[20] V. G. KAC, Torsion in cohomology of compact Lie groups and Chow rings of reductive algebraic groups, Invent. Math. 80 (1985), 69-79.

[21] N. Karpenko, Grothendieck Chow motives of Severi-Brauer varieties, St. Petersburg Math. J. 7 (1996), 649-661.

[22] N. Karpenko, A. Merkurjev, Canonical $p$-dimension of algebraic groups, $A d v$. Math. 205 (2006), 410-433.

[23] I. Kersten, U. Rehmann, Generic splitting of reductive groups, Tohoku Math. J. 46 (1994), 35-70.

[24] M.-A. Knus, A. Merkurjev, M. Rost, J.-P. Tignol, The book of involutions, AMS Colloquium Publ. 44 (1998).

[25] B. Köck, Chow motif and higher Chow theory of $G / P$, Manuscripta Math. 70 (1991), 363-372.

[26] Y. Manin, Correspondences, motives and monoidal transformations, Math. USSR Sbornik 6 (1968), 439-470.

[27] A. Merkurjev, Rost invariants of simply connected algebraic groups, in Cohomological invariants in Galois cohomology, Univ. Lecture Ser. 28, Amer. Math. Soc., 2003, 101-158.

[28] A. Merkurjev, I. A. Panin, A. R. Wadsworth, Index reduction formulas for twisted flag varieties. I, $K$-Theory 10 (1996), 517-596.

[29] M. Mimura, H. Toda, Topology of Lie groups. I, II, Translations of Mathematical Monographs 91, Amer. Math. Soc., 1991.

[30] S. Nikolenko, N. Semenov, K. Zainoulline, Motivic decomposition of anisotropic varieties of type $\mathrm{F}_{4}$ into generalized Rost motives, to appear in $J$. of $K$-Theory.

[31] I. A. PAnin, On the algebraic $K$-theory of twisted flag varieties, $K$-Theory 8 (1994), 541-585.

[32] M. Rost, The motive of a Pfister form, preprint http://www.mathematik.

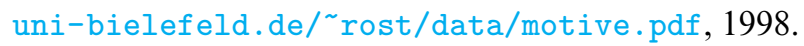

[33] M. Rost, On the basic correspondence of a splitting variety, preprint http://www . mathematik. uni-bielefeld.de/ ${ }^{\sim}$ rost/data/bkc-c.pdf, 2006.

[34] J. Tits, Classification of algebraic semisimple groups, in Algebraic Groups and Discontinuous Subgroups (Proc. Sympos. Pure Math., Boulder, Colo., 1965), Amer. Math. Soc., 1966, 33-62.

[35] A. Vishik, On the Chow groups of quadratic Grassmannians, Doc. Math. 10 (2005), 111-130.

$4^{\mathrm{e}}$ SÉRIE - TOME $41-2008-\mathrm{N}^{\mathrm{o}} 6$ 
[36] A. Vishix, Fields of $u$-invariant $2^{r}+1$, in Algebra, Arithmetic and Geometry, Manin Festschrift, Birkhäuser, 2007.

[37] V. Voevodsky, On motivic cohomology with $\mathbb{Z} / l$-coefficients, preprint http://www. math.uiuc.edu/K-theory/0639/post_mot.pdf, 2003.

[38] K. Zainoulline, Canonical p-dimensions of algebraic groups and degrees of basic polynomial invariants, Bull. Lond. Math. Soc. 39 (2007), 301-304.

Viktor PETRov
PIMS
Mepartment of Mathematical and Statistical Sciences
University of Alberta
Edmonton, AB T6G 2G1, Canada
E-mail: vpetrov@math.ualberta.ca
Nikita SEMENOV
Mathematics Institute
University of Munich
Theresienstraße 39
D-80333 München, Germany
E-mail: Nikita.Semenov@mathematik. uni-
muenchen.de
Kirill ZAINOULLINE
Mathematics Institute
University of Munich
Theresienstraße 39
D-80333 München, Germany

(Manuscrit reçu le 15 janvier 2008; accepté, après révision, le 3 juin 2008.) 